\title{
EchoGéo
}

45 | 2018

Déclinaisons géographiques du changement social en Iran

\section{Le rôle des séries télévisées turques dans la circulation des modèles urbains et des modes d'habiter en Iran}

Mina Saïdi-Sharouz

(2) OpenEdition

Journals

Édition électronique

URL : https://journals.openedition.org/echogeo/15985

DOI : 10.4000/echogeo.15985

ISSN : 1963-1197

Éditeur

Pôle de recherche pour l'organisation et la diffusion de l'information géographique (CNRS UMR 8586)

Référence électronique

Mina Saïdi-Sharouz, «Le rôle des séries télévisées turques dans la circulation des modèles urbains et des modes d'habiter en Iran », EchoGéo [En ligne], 45 | 2018, mis en ligne le 05 novembre 2018, consulté le 10 août 2021. URL : http://journals.openedition.org/echogeo/15985 ; DOI : https://doi.org/ 10.4000/echogeo.15985

Ce document a été généré automatiquement le 10 août 2021.

EchoGéo est mis à disposition selon les termes de la licence Creative Commons Attribution - Pas d'Utilisation Commerciale - Pas de Modification 4.0 International (CC BY-NC-ND) 


\title{
Le rôle des séries télévisées turques dans la circulation des modèles urbains et des modes d'habiter en Iran
}

\author{
Mina Saïdi-Sharouz
}

1 Les relations commerciales entre l'Iran et la Turquie connaissent une longue histoire. Des facteurs importants comme la proximité géographique et l'accessibilité facilitée grâce à des coûts intéressants pratiqués par les compagnies aériennes, l'exemption mutuelle du visa d'entrée et de nombreuses similitudes culturelles (les traditions, la nourriture, les valeurs, la religion et enfin la langue pour les Azéris d'Iran) ont incontestablement facilité les échanges. Cependant, depuis les sanctions imposées contre l'Iran par les pays membres de l'OCDE dont la Turquie en 2007, les échanges commerciaux officiels se sont limités et c'est par le bas et de manière informelle que se sont progressivement restructurées les relations commerciales et culturelles ${ }^{1}$. Le commerce informel et le «trabendo » ou commerce à la valise (Adelkhah, 2007; De Tapia, 2007 ; Péraldi, 2016 ; Pérouse, 2002), très bien organisés en Turquie, deviennent une voie importante de l'exportation de marchandises vers l'Iran. En dehors des hommes et des femmes d'affaire ainsi que des professionnels de commerce, de nombreux citoyens issus des classes moyennes et populaires participent à leur façon et à l'échelle micro à ces échanges (Moghadam et Weber, 2015).

2 Un autre lien important contribuant à renforcer les liens commerciaux de manière indirecte est le secteur de l'audiovisuel, et tout particulièrement l'industrie des séries turques, renouvelant les relations culturelles et économiques entre les deux pays. Depuis 1979, suite à la censure de la télévision iranienne après l'avènement de la République islamique, les téléspectateurs iraniens se sont tournés vers les chaînes étrangères, dont les chaînes turques, diffusées par satellite. Ces diffusions ont joué un rôle important dans la promotion du secteur touristique, mais aussi dans la circulation de modèles et de représentations en Iran. En dehors de la mise en valeur du patrimoine 
matériel, le «modèle turc » véhicule les valeurs morales et les identités nationales de la nouvelle Turquie de l'AKP. Un modèle qui se présente comme un idéal de gouvernance où coexistent harmonieusement l'économie de marché et la tradition musulmane dans un cadre démocratique. En d'autres termes, les séries turques donnent à voir comment la Turquie revendique de concilier les valeurs de l'Islam et les libertés individuelles (Massicard, 2012). Cependant, certains gouvernements des pays de la région, dont la République islamique d'Iran, critiquent le contenu de telles émissions car véhiculant à leurs yeux des traditions et mœurs aliénantes et insuffisamment conformes à l'Islam.

Les chaînes de télévision turques ont investi beaucoup de moyens financiers pour conquérir le marché iranien et faire concurrence aux chaînes arabes diffusées largement dans la région. Si les chaînes arabes ont été très regardées après la révolution en Iran, surtout grâce à leurs émissions en direction des femmes autour du tricot, de la cuisine et des films comiques, les chaînes turques leur ont volé la vedette dans les années 2000 et ceci en grande partie grâce aux séries télévisées².

Dans nos travaux antérieurs, nous avions montré la relation existant entre les séries turques et le commerce du prêt-à-porter en Iran en analysant la manière dont les séries contribuent à créer de la demande pour l'importation des marchandises turques. Nous avions également étudié le rôle des touristes dans l'importation des marchandises valorisées dans les séries turques (Saïdi-Sharouz, 2015). Dans le présent travail, nous cherchons à comprendre le rôle des séries télévisées turques (interdites en Iran mais diffusées par satellite sur les chaines étrangères) dans l'architecture et le mode d'habiter des Iraniens ainsi que dans les modèles urbains. Nous interrogerons ainsi le rôle des séries turques à grand succès (telles que Le siècle magnifique, L'amour interdit et Bargrizan) dans le transfert des modèles urbains et architecturaux et des modes d'habiter en Iran. La circulation des modèles est étudiée à plusieurs échelles (espace domestique, géographie commerciale de la ville et production de grands complexes résidentiels). Nous examinons également la manière dont les séries contribuent à faire circuler des modèles dans le domaine du décor et de l'architecture entre les deux pays. Comment les produits culturels turcs et notamment les séries télévisées servent-ils de socle à l'amplification des échanges commerciaux avec l'Iran et au transfert de modèles urbains mondialisés dans une République islamique?

Afin de mesurer l'importance de ce phénomène, nous avons mené une enquête de terrain de type qualitatif en Iran entre 2016 et 2017, grâce à plus de quarante entretiens approfondis avec des spectateurs des séries, d'âges et classes sociales variés, habitant pour la plupart à Téhéran. Dans le choix des personnes, nous avons fait appel à notre cercle d'interconnaissance mais aussi à des profils plus variés à travers des réseaux éloignés (les populations des quartiers modernes et populaires). Nos questions étaient ciblées sur le temps passé à regarder les séries, les préférences au niveau des séries, les éléments architecturaux retenus et enfin les changements intervenus dans le comportement, le mode d'habiter et la décoration grâce aux séries. Il faut préciser que $70 \%$ des enquêtés sont des femmes entre 18 et 90 ans car ce sont les femmes qui constituent l'essentiel du public des séries et qui sont également chargée de la décoration des logements. Nous avons également mené des entretiens avec des couples ou une famille entière afin de susciter un débat entre les générations et les sexes. Ce type d'enquête - qui s'approche de l'entretien auprès d'un groupé ciblé (focus group), a été très utile pour recueillir les avis mais aussi pour mesurer les impacts des séries sur le mode de vie familial. Nous avons visité leurs appartements et nous avons, à de 
nombreuses reprises, regardé les séries avec les personnes enquêtées afin de collecter directement leurs réactions. Nous avons également mené une enquête dans les centres commerciaux et les lieux de vente du mobilier et de l'immobilier. Enfin, nous avons étudié un corpus d'une trentaine de séries télévisées les plus regardées en Iran et plus particulièrement cinq séries significatives ${ }^{3}$.

Nous considérerons tout d'abord le rôle important des séries turques dans le transfert culturel, celles-ci créant un filtre culturel entre les films occidentaux et les spectateurs iraniens. Dans un second temps, nous chercherons à comprendre la manière dont les séries télévisées turques créent du désir et transforment les modes de vie en Iran. Ensuite, nous montrerons comment ces séries contribuent à une forme de turquisation de la société iranienne. Nous évoquons également, à travers des exemples, la circulation de modèles architecturaux turques (design, mobilier, objet, etc.) en Iran et enfin nous verrons comment l'imagerie des séries crée un socle favorable aux échanges économiques, notamment dans le domaine de la construction et de l'immobilier.

\section{Les chaînes turques, un filtre culturel entre l'Iran et l'Occident}

7 Comme d'autres pays émergents (notamment en Asie ou en Amérique latine), la Turquie est devenue un pôle émetteur de contenus médias. À l'image de l'exportation culturelle des grandes puissances (Nye, 1990 ; Öktem, Abou el-Fadl, 2009), le soft power turc permet ainsi un transfert de modèles économiques et culturels vers d'autres pays. En 2009 la Turquie décide de renforcer ses relations économiques, politiques et culturelles avec les États de la zone MENA (Middle East North Africa, Moyen-Orient et Afrique du Nord) (Tutal-Cheviron et Çam 2017). Cette nouvelle stratégie traduit l'ambition affichée par le gouvernement AKP d'élever la Turquie au rang de puissance régionale et de "modèle $»^{4}$. Ces orientations/politiques impulsées par le gouvernement AKP sont mises en œuvre à l'aide du réseau public de télévision et de radio (TRT), à travers ses productions médiatiques (informations, séries et films).

En tant que "modèle » pour la plupart des pays musulmans, la Turquie exerce une fonction de filtre culturel. Les feuilletons turcs sont vus comme des produits culturels qui prendraient davantage en compte que ceux de "l'Occident» les spécificités des histoires sociales, politiques et religieuses. Dans une enquête réalisée en 2011 sur un échantillon de 2323 personnes par TESEV 5 dans 16 pays du monde arabe et musulman, $78 \%$ des personnes interrogées avaient une perception positive de la Turquie, $74 \%$ reconnaissaient avoir regardé au moins une fois dans leur vie une série turque, et la plupart d'entre elles étaient capables de nommer des séries turques et leurs acteurs. (Tutal-Cheviron et Çam, 2017). Le Directeur général de la TRT déclarait que jusqu'à ce que le phénomène de la série turque apparaisse, la Turquie avait mauvaise réputation dans la région arabe, mais que, par la suite, la perception des Turcs et de la Turquie avait changé : "Peut-être la Turquie ne génère-t-elle pas un revenu élevé grâce aux séries mais il n'y a pas de prix pour transférer notre culture et nos structures sociales sous forme de soft power à l'étranger à travers les séries ${ }^{6}$.

9 Selon le chercheur français Julien Paris, «les succès d'audience des séries turques recouvrent plusieurs réalités, à la fois en termes d'influence culturelle de la Turquie et de stratégies commerciales et économiques de la production audiovisuelle turque. Les 
chiffres du box-office cachent aussi des différences profondes dans la réception de ces séries parmi les fragments distincts constitutifs des populations de la région » (Paris, 2013). En Iran, les séries turques ont connu leur apogée depuis cinq ans avec la série culte Harim-é-Sultan ( Le siècle magnifique »), sur la vie dans le harem du Sultan Ahmed. Puis, les séries telles que Ezal, Eshgh-é Memnu' ${ }^{7}$ Laley Devri et Bargrizan ont connu une forte audience. Il est difficile d'avoir des chiffres exacts sur l'audience, car ces diffusions ne sont pas reconnues officiellement par l'Iran. Mais des études effectuées par ISPA (le centre universitaire du sondage d'opinion en Iran) ${ }^{8}$ ont estimé le nombre de spectateurs réguliers des séries télévisées à plus de 20 millions de personnes en Iran en 2017.

\section{Comment les séries télévisées turques créent-elles du désir et transforment les modes de vie en Iran?}

10 Depuis 2006, le GEM TV, la chaîne de télévision en langue persane implantée à Dubaï et à Istanbul ${ }^{9}$, interdite en Iran, diffuse par satellite les séries achetées à la télévision turque en les doublant en persan. Elle a progressivement récupéré l'audience des chaînes turques ${ }^{10}$. Cette chaîne est considérée par les autorités iraniennes comme une chaîne subversive. Elle concurrence deux autres chaînes iraniennes diffusées par satellite, dont Parsi 1, et la chaîne Manoto dont l'audience ne fait qu'augmenter depuis 2012. La télévision iranienne déploie en revanche de grands moyens pour produire des séries locales dont la plus populaire est la série Shahrzad qui connait un grand succès grâce à une liberté relative au niveau des mœurs ${ }^{11}$. Cependant, les résultats restent mitigés à cause de la moralisation et des lignes rouges définies par l'Etat, encore trop strictes pour le goût des spectateurs.

11 Les Iraniens ont aussi adopté les séries turques au détriment des novelas latinoaméricaines à grand succès, car ils se sentent plus proches de ce que les scénaristes turcs cherchent à renvoyer comme image de leur pays. Les séries sont conformes aux valeurs religieuses et traditionnelles, tout en donnant à voir le côté moderne et progressiste de la Turquie. En effet, les séries les plus populaires en Iran mettent l'accent sur les valeurs et les coutumes qui concernent les structures familiales, les relations entre les hommes et les femmes et entre les générations. Elles montrent, d'un côté, les travers d'une société traditionnelle et les souffrances que celles-ci peuvent entraîner pour les individus en les privant de leurs désirs et, de l'autre, des individus modernes à la dérive et en perte de repères et d'identité. Face à un tel tiraillement, l'option proposée par les séries est la possibilité de dialogue entre les générations. L'image d'une Turquie qui réussit à concilier les traditions et la modernité attire de plus en plus d'Iraniens en quête d'un cadre démocratique et ouvert sur le monde. De manière générale, les publicités s'appuient sur les lieux et les paysages utilisés dans la scénographie des films et contribuent à produire du rêve comme une échappatoire à la réalité et à la difficulté de vivre sous l'embargo international.

Ce qui nous semble important à soulever est que ces séries véhiculent un modèle urbain globalisé et promeuvent un certain luxe démocratisé. Les voitures de haut de gamme, les cafés branchés, les centres commerciaux pour VIP et le paysage grandiose d'une métropole en pleine croissance font rêver les Iraniens devant leur petit écran. 
13 Nos enquêtes auprès des jeunes spectateurs de séries nous révèlent leur désir de rompre avec les restrictions économiques et l'austérité dans l'Iran postrévolutionnaire. La liberté de choix et la variété des formes et des couleurs proposées par les séries représentent pour certains une forme de résistance à cette austérité. « La Turquie est aujourd'hui ce que l'Iran allait être s'il n'y avait pas eu de révolution... » observe avec regret un spectateur interrogé sur les séries. Les images médiatisées jouent un rôle important dans la construction d'un imaginaire collectif différent de celui proposé par les médias iraniens. Elles permettent aux spectateurs de recevoir, d'interpréter et de retenir les éléments du quotidien d'un univers différent.

Le GEM TV, grâce à sa forte audience, est devenue une chaîne promotionnelle pour les produits commerciaux turcs. Les séries turques « iranisées ${ }^{12}$ » sont diffusées tout au long de la journée. Elles sont entrecoupées de spots publicitaires en direction des Iraniens voulant voyager ou investir en Turquie dans le secteur du bâtiment. Les spectateurs s'approprient les objets et les décors qui leur deviennent familiers grâce à une conception scénographique efficace. Le décor des séries utilise des objets et des vêtements de fabrication turque et familiarise les spectateurs à l'univers des personnages et à leur mode de vie. Ainsi les scènes de fiction deviennent des références, une source d'apprentissage de modèles sur la façon de résoudre des problèmes et de gérer des situations difficiles (Comstock et al., 1978 cité par Comte 2013 ; Novak, 1981; Esslin, 1982). Certains auteurs considèrent que le divertissement apporté par les séries est non seulement source d'équilibre, mais entraîne également l'individu à gérer les difficultés que la vie peut engendrer et que les séries développent au fil des épisodes. Ces séries constituent ainsi une sorte de "source de guide de comportement» (Comte, 2013), à l'image de celui que les parents, l'Église ou la communauté pouvaient offrir, avec une liberté interprétative des messages reçus puisqu'elles co-construisent leur perception avec leur «capital culturel ». Aux ÉtatsUnis, des psychanalystes sont allés jusqu'à attribuer un rôle quasiment thérapeutique aux images de fiction, ainsi à l'appui du succès des séries (Comte, 2013).

15 Ces séries utilisent des techniques de mise en scène très efficaces pour donner à voir le cadre et les détails d'une scène. Par exemple, les plans qui se déroulent à l'intérieur d'une maison sont assez longs pour permettre aux spectateurs de faire l'inventaire des objets en vue de s'inspirer de l'architecture intérieure. Le pic dramatique, le dénouement des intrigues développées par la série sont situés dans des espaces calculés. La forme, la couleur et les détails de ces lieux restent davantage ancrée dans l'inconscient des spectateurs. Les recherches de Carmen Comte mettent en lumière la manière dont le spectateur repère les éléments qui lui paraissent importants et fait des recoupements pour accéder à une logique de l'histoire comme dans sa vraie vie." (Compte, 2013). Par ailleurs, comme le remarque Aubry, «le continuum narratif de la sérialité, imitant le flux de la temporalité réelle forme une sorte d'écho fictif de la vie quotidienne du public » (Aubry, 2006). L'idée d'adhésion et de co-construction d'une réalité qui ne dépend que de l'individu renvoie au phénomène de mimétisme. Le mimétisme pourrait être considéré comme le premier signal visible d'un impact de l'image animée, qu'il soit profond ou superficiel. (Bandura, 1977).

Par le phénomène du mimétisme, les modes de vie turcs servent de modèle pour ceux qui souhaitent être connectés au monde extérieur. Entrer dans l'économie globale à la façon turque devient progressivement un objectif pour les Iraniens en manque d'Occident. Les séries proposent une autre vie aux spectateurs : une vie meilleure qui se 
déroule dans un décor idéalisé. En s'appropriant ces scènes, les spectateurs accèdent de manière virtuelle à cette autre vie. Ainsi, les scènes deviennent des références mentales auxquelles les individus peuvent se référer. Le décor, le lieu et le paysage montrés dans les séries, mêlant une image traditionnelle orientale à la modernité occidentale, restent ancrés dans la mémoire de celui qui les regarde. Sur ce même principe, les téléspectateurs cherchent à imiter les acteurs et s'inspirent des éléments décoratifs des séries pour concevoir leurs intérieurs. Servent par exemple de modèles la chambre à coucher de Khorram Sultan, de style ottoman, ${ }^{13}$ dans la série Le siècle magnifique, une série historique, à grand succès, le salon d'Asma Sultan et le bureau des frères Buran, riches commerçants de Bursa, dans La mariée d'Istanbul ${ }^{14}$. De manière générale, le mobilier turc importé ou fabriqué sur place, s'inspire d'un mélange de styles, dont le style ottoman, le néo-classique éclectique (Styles arménien, ottoman et européen) ${ }^{15}$, le néo-oriental, l'Art nouveau et le style moderne international (Pinon, 2006).

Depuis quelques années, grâce à l'importation des produits turcs sur le marché, le spectateur iranien peut enfin accéder concrètement à ces décors. Progressivement, un changement de goût se généralise par effet de contagion (Tarde, 1890) dans les réseaux de sociabilité et la mode de ces objets se propage dans le pays. Ainsi, les intérieurs deviennent une image en soi permettant aux maître et maitresse de maison de produire un message à destination de leurs hôtes : leur agencement suit un impératif participant à une quête de prestige. Les personnes enquêtées nous confirment à quel point elles font attention aux jugements des autres concernant leur aménagement intérieur. L'ambiance et l'aménagement intérieur (choix du style, de la couleur, des matériaux, etc.) vus dans les séries deviennent la référence du bon goût du moment.

Illustration 1 - Le mobilier de luxe « vu à la télévision »

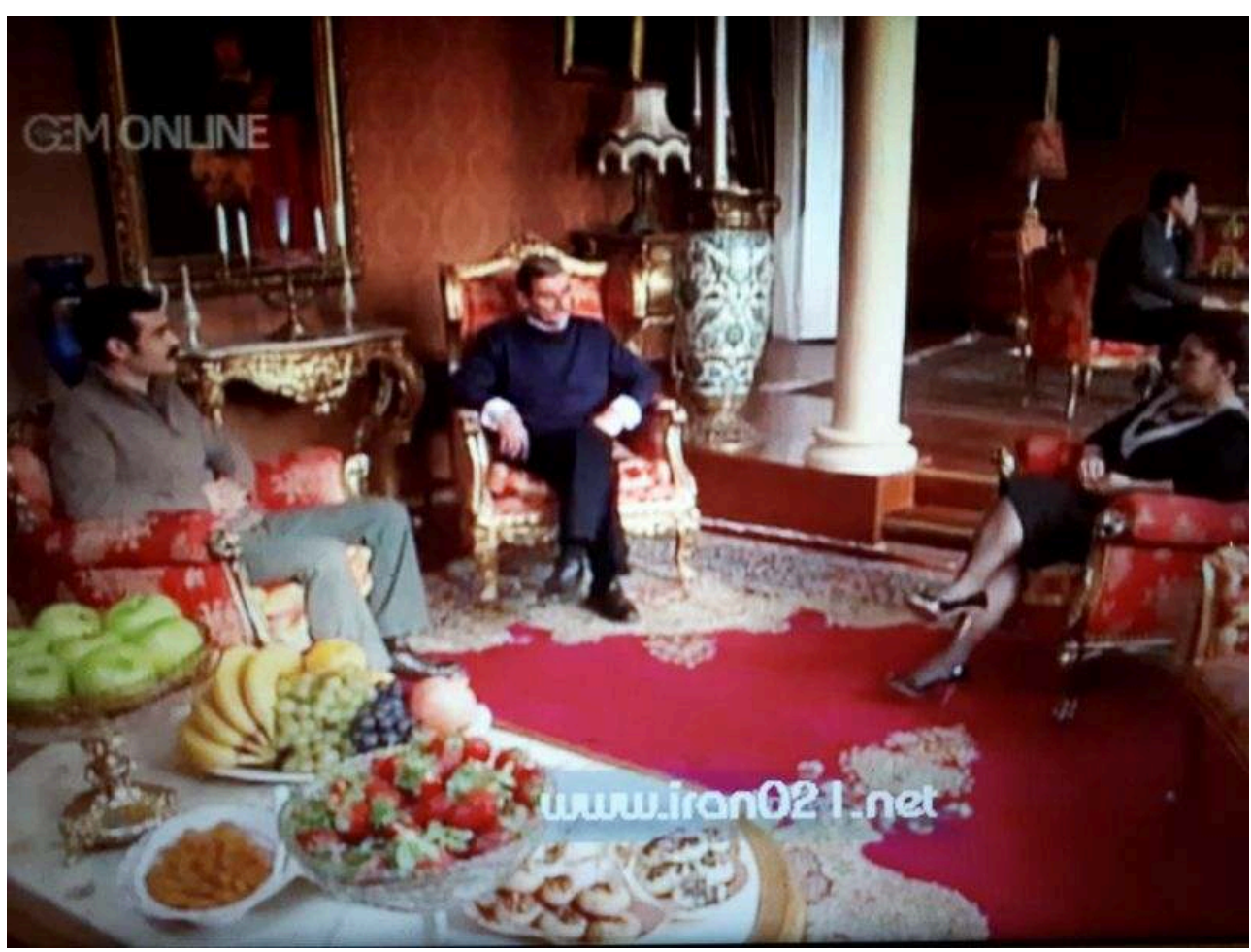

Villa de luxe dans la série Bargrizan.

Auteur : capture d'écran, Mina Saïdi-Sharouz. 
Illustration 2 - Le mobilier de luxe exposé dans les magasins de Téhéran

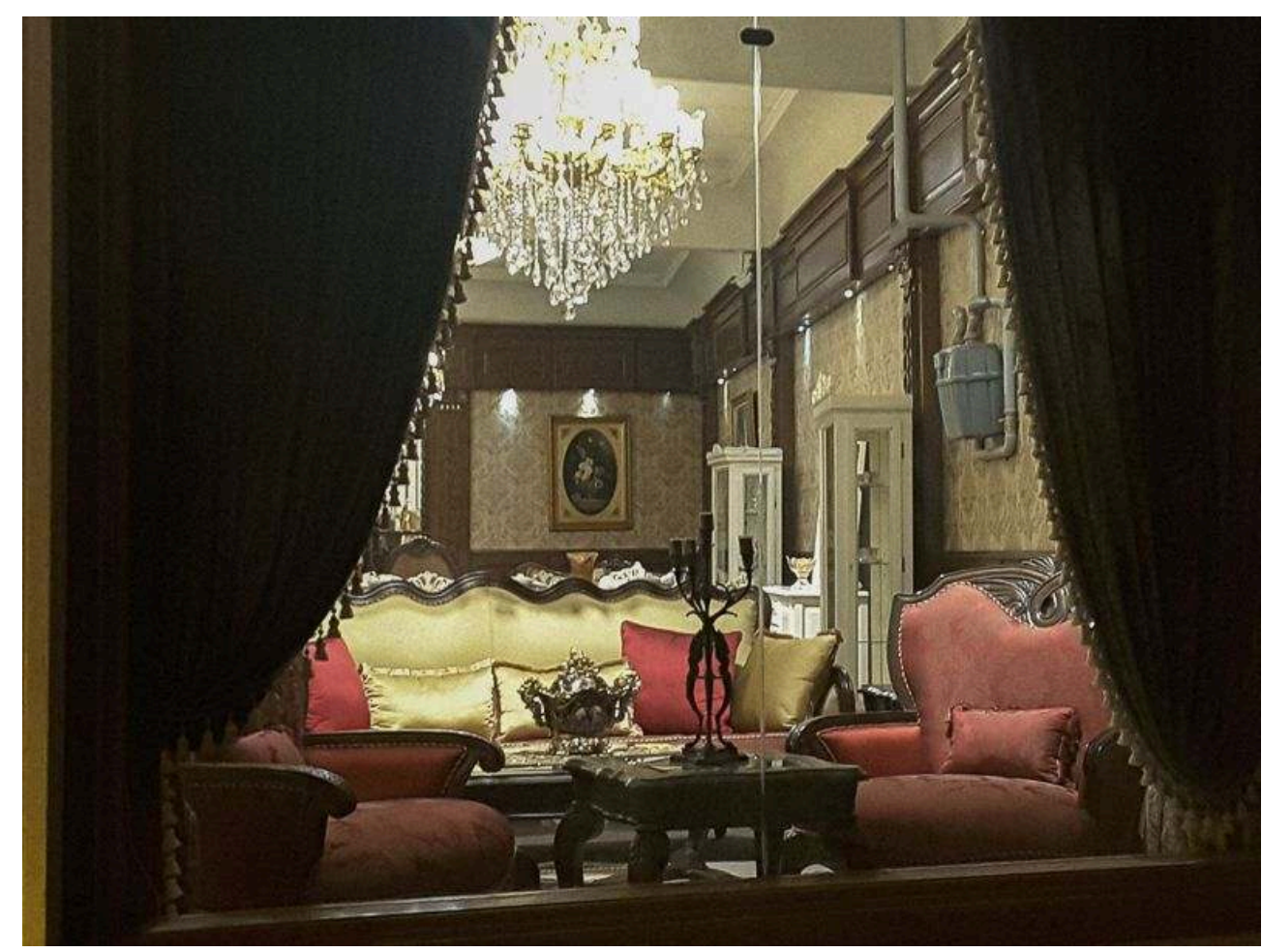

Un magasin de mobilier turc à Téhéran avec un mobilier chic et contemporain.

Auteur : Mina Saïdi-Sharouz.

\section{Une affaire de goût}

Les modèles véhiculés tiennent compte des différences sociales. Notre étude montre comment les différentes catégories sociales sont représentées dans les séries, à travers une mise en scène finement réfléchie des lieux investis par les personnages des films. Le public iranien, en s'identifiant aux personnages des fictions, cherche dès lors à imiter leurs comportements et leurs modes d'habiter. Les espaces et les décors dans lesquels ils agissent prennent alors une signification particulière. Par exemple, toute la famille élargie des Buran, dans la série La mariée d'Istanbul ${ }^{16}$ cohabitent dans un « emârat " ${ }^{17}$. Omar, jeune créateur de mode dans L'amour loué (Eshghe Ejarei) ${ }^{18}$, habite un grand duplex moderne au design international comme dans toutes grandes métropoles, avec des objets et un mobilier contemporains mondialisés et anonymes (canapé en cuir cossu, bar à l'américain, de hauts tabourets, des tapis modernes et la peinture murale de couleur sombre éclairée par des spots halogènes). Chaque détail est minutieusement étudié. Au petit déjeuner, Omar prend un espresso avec une machine à capsules, tandis que sa bien-aimée, issue d'un milieu plus modeste, continue à prendre du thé turc préparé sur un samovar ${ }^{19}$. Ainsi, l'alliance entre l'identité turque et l'appartenance à l'économie mondiale se met en scène à travers des objets quotidiens. 
Illustration 3 - Le design contemporain dans la série Amour Loué

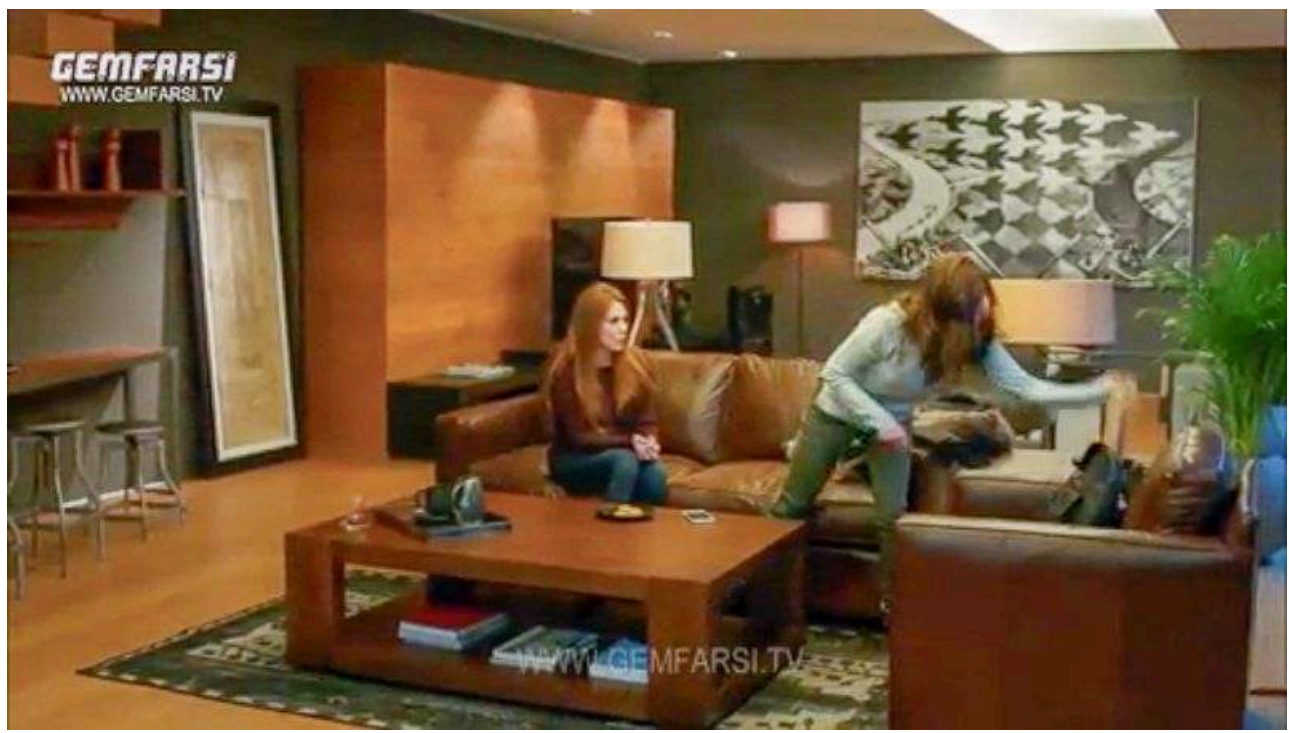

Daphné et Omar dans l'appartement très moderne de ce dernier.

Source : GEM TV.

\section{Illustration 4 - Le design contemporain Bargrizan}

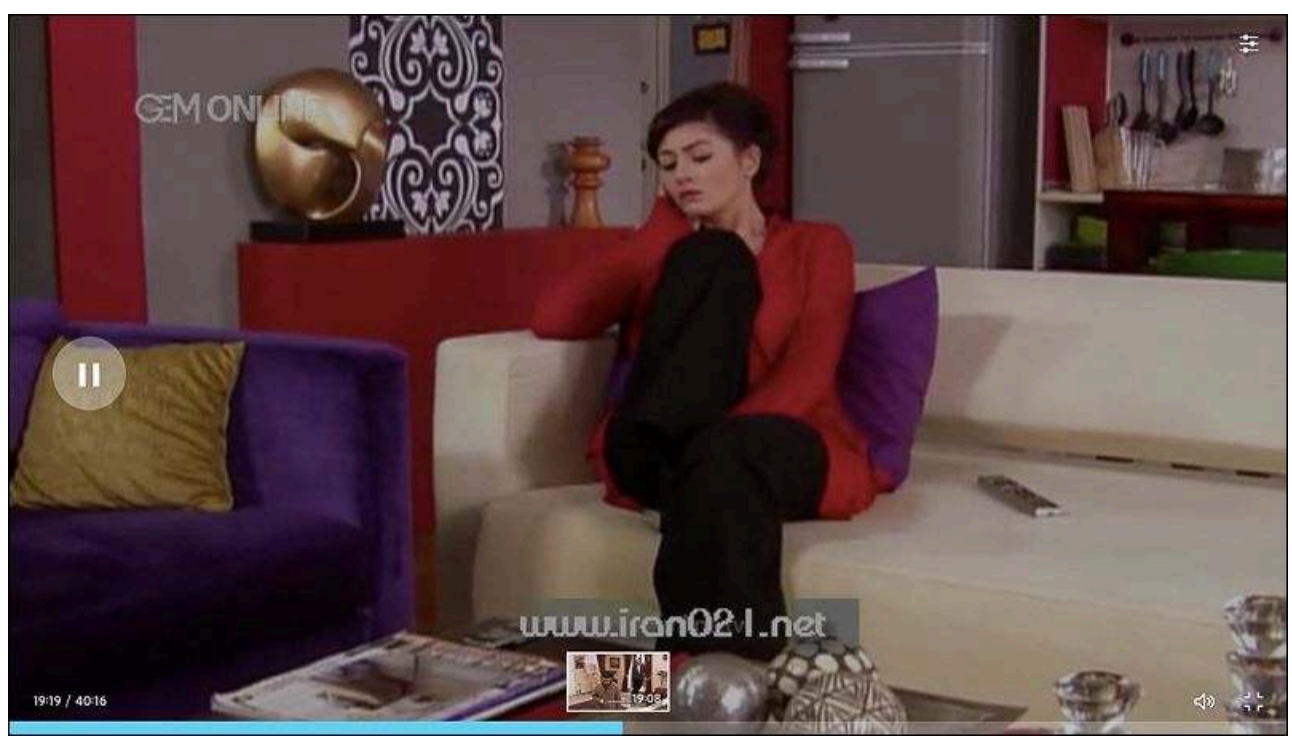

Farkhondeh, jeune fille célibataire, modèle d'une femme moderne peu attachée aux valeurs anciennes, change plusieurs fois d'appartement et de mobilier au cours de la série.

Source : GEM TV. 
Illustration 5 - Le style « nouveau riche »

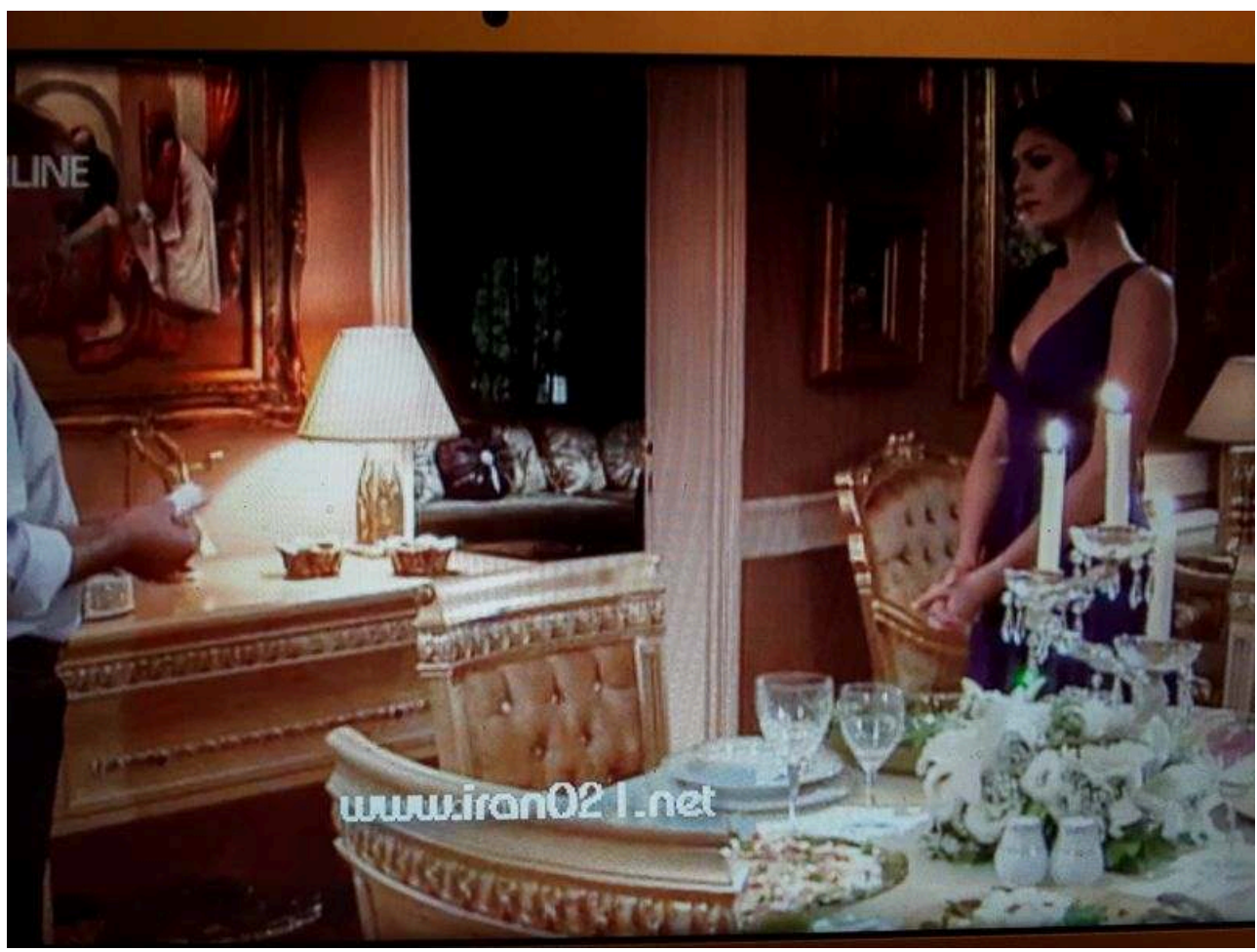

Source : Série Bargrizan

Les lieux de vie des personnages sont sciemment choisis. Dans les séries d'action, comme Karadayi ${ }^{20}$, le héros du film et sa famille habitent un quartier populaire et chaleureux tandis que les gangsters séjournent dans des ruines sans âme. Des chefs de famille intègres et attachés aux valeurs morales comme dans Barguerizan ${ }^{21}$, habitent des appartements ou maisons en bois de style ottoman, représentées comme traditionnelles et authentiques. Les personnages négatifs des films habitent dans des grandes villas bourgeoises avec un décor de style rococo et kitch, rappelant l'époque impériale (dorures, meubles massifs et chandeliers). Bref, un univers de nouveaux riches sans goût, à l'opposé des familles nobles et authentiques qui habitent les emârats familiaux.

La ville est souvent montrée de manière binaire. Les quartiers d'affaires et les gated communities $^{22}$ où circulent les voitures de luxe s'opposent aux quartiers populaires exotiques, avec des enfants jouant dans la rue, un café traditionnel, des artisans locaux, des rues piétonnes. Si les quartiers modernes reflètent la réussite économique et, de ce fait, la concurrence et les rivalités engendrées par la croissance et l'innovation technologique, dans les quartiers populaires, les relations de voisinages sont données à voir de manière idyllique, évoquant la nostalgie d'un passé révolu qu'on cherche à conserver à travers les images reconstituées.

\section{L'importation du mobilier et des éléments de décor vus à la télévision}

21 En s'appuyant sur une demande croissante des Iraniens pour l'ameublement et la décoration intérieure "vus à la télévision", de nombreux commerçants iraniens se 
sont consacrés à l'importation des meubles et des accessoires de maison depuis la Turquie. À Téhéran, par exemple, depuis une dizaine d'années, des zones entières de la ville se sont spécialisées dans la vente des meubles et des éléments de décoration intérieure turcs comme les rideaux, les luminaires, etc. ${ }^{23}$ Les marchandises importées de Turquie et acheminées de manière illicite sont exposées et vendues dans les espaces privés mais aussi dans les magasins en créant progressivement des zones spécialisées. Il s'agit d'une articulation des secteurs formel et informel. Par exemple, les magasins de rideaux turcs se placent côte à côte et créent des « rastehs " (rues spécialisées) ${ }^{24}$ dont le long de la rue Bahar, la Place Felestin, et Vali Asr pour les plus luxueux, etc. ${ }^{25}$; les tissus d'ameublement venus de Bursa créent un autre pôle autour du quartier Polé Tchoubi et de Sohravardi.

Illustration 6 - Carte des zones de produits turcs à Téhéran

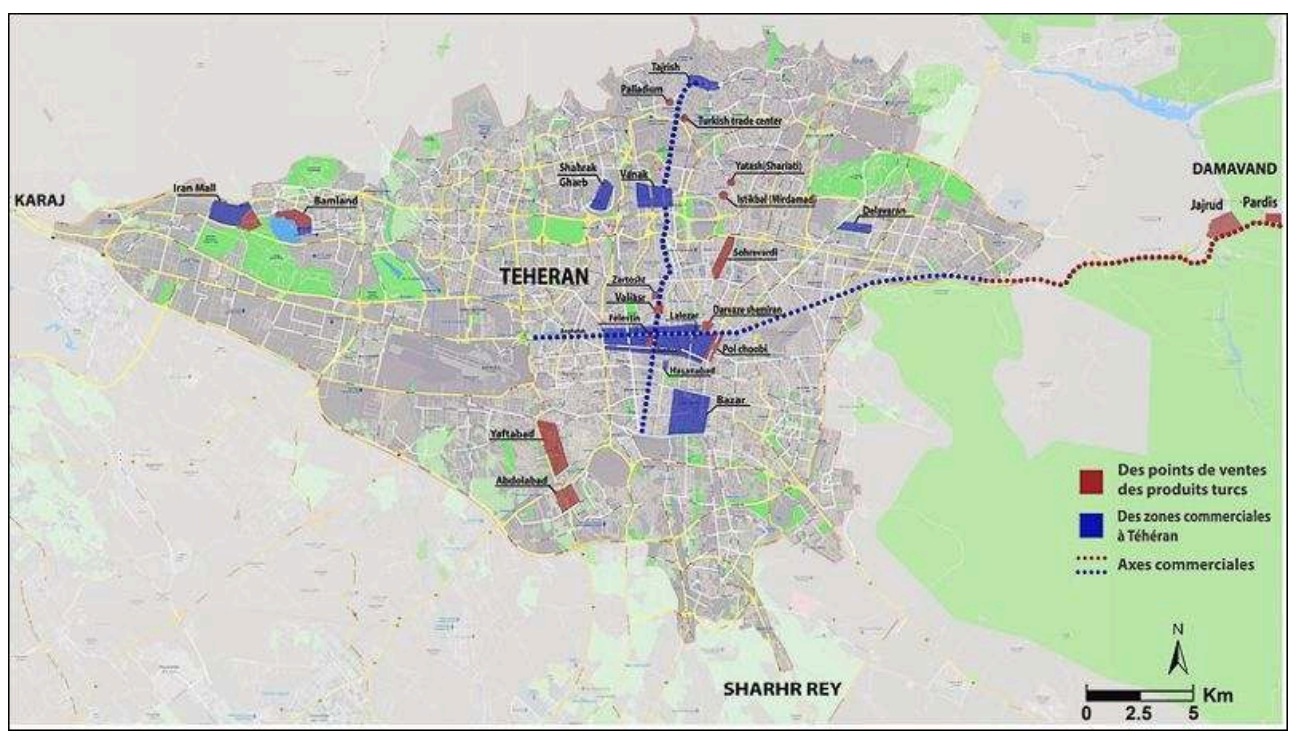

Auteur : Mina Saïdi-Sharouz, juin 2018.

Le phénomène le plus spectaculaire réside cependant dans l'occupation de l'espace par l'exposition et la vente des meubles turcs importés ${ }^{26}$. Avec l'arrivée des meubles turcs sur le marché, les surfaces de vente s'étalent et se multiplient. Les lieux de vente se séparent des lieux de fabrication. Par exemple, l'ancien quartier des meubles, Yaftabad, situé au sud de Téhéran, s'est rapidement spécialisé dans les produits de luxe turcs et les ateliers de fabrication se déplacent vers l'extérieur de la ville ${ }^{27}$. Aujourd'hui, une nouvelle zone commerciale se forme autour de ces ateliers et compte plus de 150 grands salons d'exposition et de vente dont une grande partie de l'achalandage est constituée de meubles turcs. Afin d'attirer la clientèle, les magasins exposent des meubles à différents prix. Des meubles turcs sont juxtaposés aux meubles iraniens. Certains magasins exposent du mobilier fabriqué en Iran mais s'inspirant fortement des marques turques telles que Istiqbal, Safak ou Enza Home ${ }^{28}$, permettant aux clients peu fortunés de les acquérir à un prix beaucoup plus accessible (jusqu'à un tiers du prix des meubles importés). 
Illustration 7 - Espaces d'exposition et de vente du mobilier turc à Téhéran

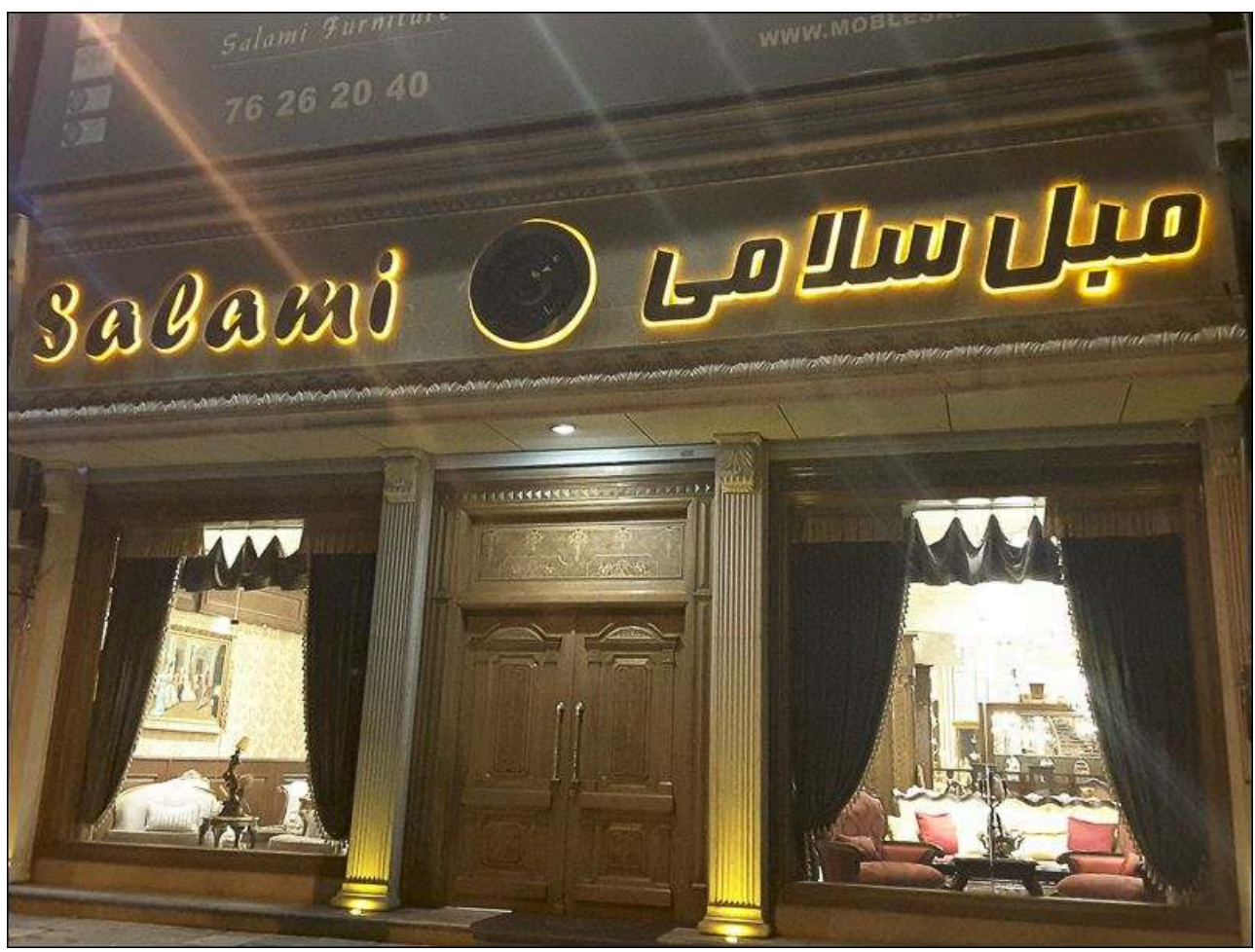

Un magasin exposant d'un côté (droite) des meubles turcs importés et d'un autre côté (gauche) des meubles fabriqués en Iran à moindre coût.

Auteur : Raana Anarchi, novembre 2017

$\mathrm{Si}$, traditionnellement, les clients achetaient les objets un par un dans différents quartiers de la ville, désormais ils trouvent quasiment tous les objets " vus à la télé " dans le même magasin. Ils peuvent acheter un salon entier au détail près, identique à celui d'une série. Une enquêtée de 45 ans, mère de famille et résidente à Machhad, nous explique : «Au départ je m'intéressais aux vêtements et surtout aux coiffures dans les séries car il n'y avait pas de restriction et on pouvait voir comment les autres femmes étaient coiffées. En regardant les scènes d'intérieur, j'ai fini par observer les accessoires et maintenant, je sais que je peux les acheter dans les magasins à Machhad. Dans la rue Salman il y a dix nouveaux magasins qui vendent les objets de la maison. Les films turcs nous inspirent pour notre décor intérieur alors que les films iraniens n'offrent pas de détails de décor et de mise en scène aussi travaillés ».

24 A Téhéran, les marques Henza Home et Istiqbal sont les deux premières grandes marques turques de fournitures pour la maison à ouvrir des boutiques. Istiqbal est un magasin d'ameublement plutôt classique alors que Henza Home propose un design plus contemporain. De plus, il bouleverse le système traditionnel des rastehs en regroupant dans le même espace tous les articles de la maison allant des meubles aux horloges murales, tapis, vaisselles et objets décoratifs très variés. Le magasin est géré par des directeurs turcs et le personnel de la vente est entièrement formé en Turquie. Depuis 2016, d'autres magasins affichant le même concept ont ouvert à Téhéran, comme la chaîne Çelik, spécialisée dans les chambres complètes pour enfants. 
Illustration 8 - Magasin turc Enza Home Yatas à Téhéran

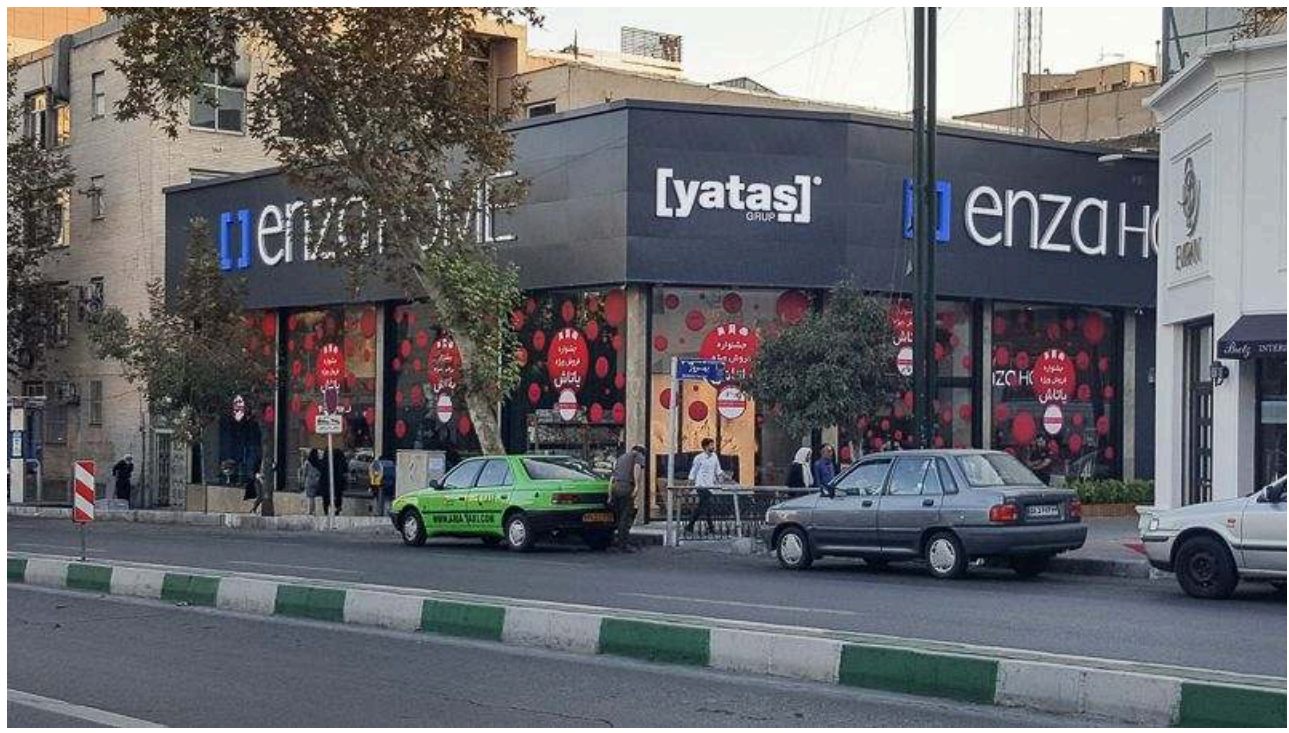

Le magasin turc Enza Home Yatas vend les objets de décor « vus dans les séries » dans le même espace.

Auteur : Mina Saïdi-Sharouz, octobre 2017.

Plus proche du goût iranien tout en étant moderne et universel, le mobilier turc répond à une variété de goûts en Iran. Du plus traditionnel au plus moderne, les fabricants de meubles choisissent les couleurs appréciées par les Iraniens. Par exemple, selon les revendeurs de meubles turcs, les clients iraniens préfèrent les couleurs sobres aux teintes plus prononcées. «On leur propose les modèles turcs avec la possibilité de changer les coloris des tissus ». Ils constatent que les jeunes commencent à avoir plus d'audace et d'originalité dans leurs choix de décoration intérieure et tentent des nouveautés pour affirmer leur singularité.

Illustration 9 - Intérieur dans une scène de film

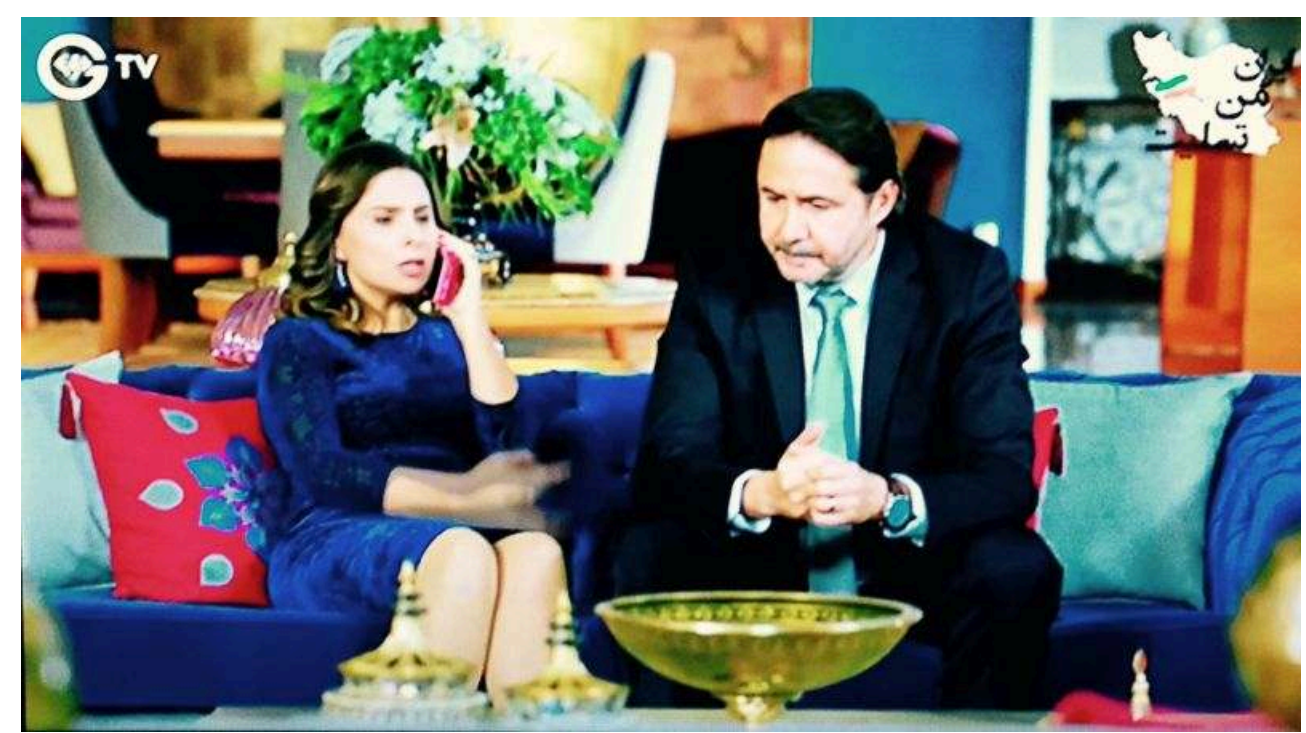

Le mobilier moderne dans la série Marhemat.

Source : Gem TV. 
Illustration 10 - Intérieur présenté au magasin Yatas

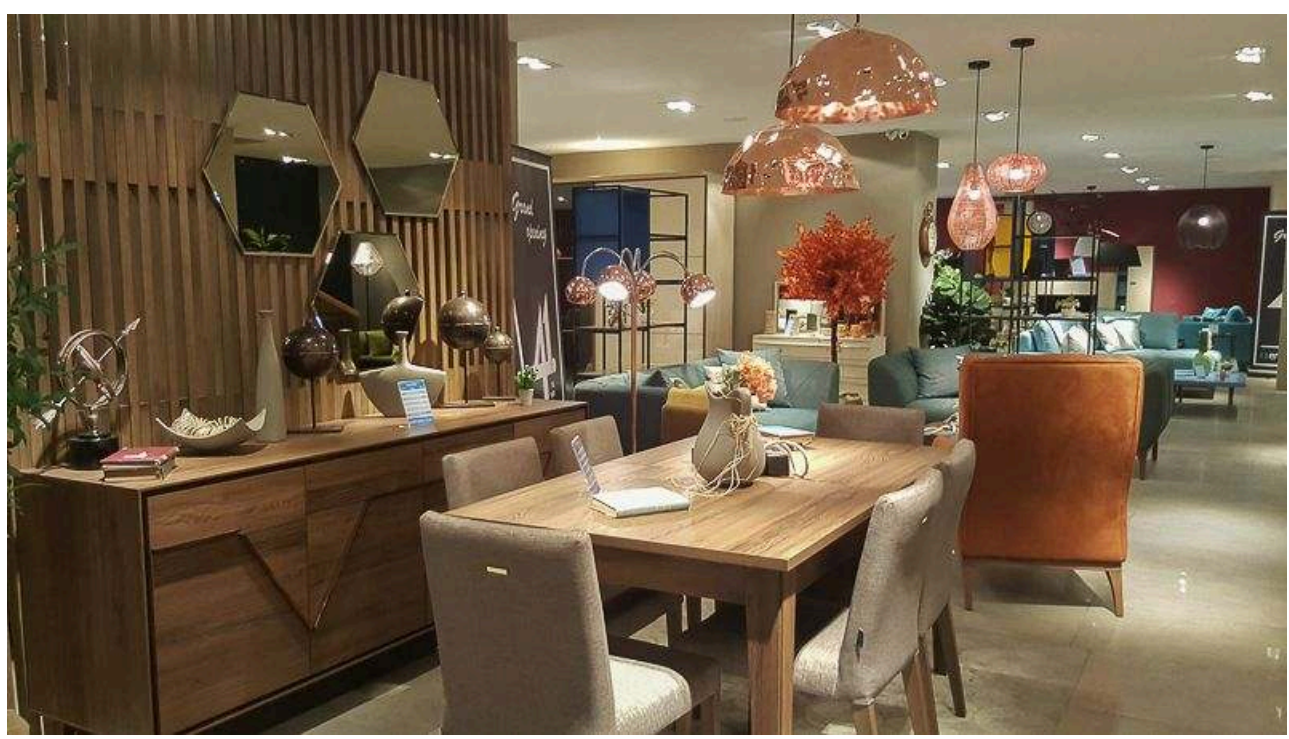

Les meubles modernes « vus dans les séries » sont vendus dans ce magasin.

Auteur : Mina Saïdi-Sharouz, octobre 2017

Une cliente d'un magasin de meubles turcs, une jeune femme de 30 ans nouvellement mariée, explique : "On préfère les meubles turcs car ils sont de plus petite taille par rapport aux meubles iraniens et arabes qui imitent les produits américains et sont faits pour des immenses salons. Nous voulons avoir de beaux meubles classiques mais plus petits en taille avec moins de dorures ». Un vendeur du magasin Yatas interrogé sur les goûts des acheteurs observe: "Nous essayons de varier les offres d'ameublement et de proposer des objets plus originaux, mais les clients iraniens sont attachés à ce qu'ils voient dans les séries. La plupart me demande un lit comme celui de Khorram sultan et je leur dis que ce n'est pas notre style. Mais, heureusement, des jeunes s'intéressent à d'autres meubles aussi. Reste qu'on peut voir rapidement ceux qui regardent les séries et ceux qui s'en fichent ».

\section{La « turquisation » de la société et le désir mimétique}

Afin de susciter la demande en créant un désir mimétique (Girard, 2008) des modes de vie et des nouvelles formes de consommation, les chaînes s'appuient aussi sur les spots publicitaires diffusés lors des séries. La publicité a aussi pour objectif de faire la promotion des produits turcs ${ }^{29}$. Par exemple, les annonces pour le prêt-à-porter « made in Turkey " tels que les jeans Collins et Mavi ou encore les chaussures Flo, pendant les séries, contribuent à les faire connaître et à créer la demande en Iran. Une fois la demande créée, les produits sont importés en Iran. La publicité préalable leur facilite largement la vente ${ }^{30}$. De plus, les génériques des films témoignent du nombre important de marques intervenant comme sponsors. Le succès de ces marques qui ne concernaient dans les années 1990 qu'une petite élite iranienne touche aujourd'hui une grande partie de la classe moyenne. Ceci grâce, en grande partie, à l'impact des séries sur les modes de consommation.

Ce phénomène ne se limite pas à l'architecture intérieure. En ce qui concerne le domaine de l'immobilier, les publicités diffusées pendant les séries se multiplient pour 
présenter des appartements à vendre à Istanbul ou sur le littoral turc avec des décors luxueux et des vues imprenables sur le Bosphore ou sur la Méditerranée. Un bandeau d'annonce pour les appartements de luxe est déroulé à répétition en bas de l'image. Les sociétés immobilières vont jusqu'à proposer l'obtention d'un passeport européen aux futurs propriétaires. D'autres annonces publicitaires présentent des sociétés intermédiaires aidant les Iraniens à émigrer vers la Turquie, obtenir une carte de séjour et ouvrir un compte dans une banque internationale ${ }^{31}$. Ces publicités viennent confirmer au public des séries qu'il est possible d'accéder à une autre vie et de devenir propriétaire en Turquie avec un prix abordable ${ }^{32}$.

Illustration 11 - La publicité diffusée lors des séries pour la vente d'appartements en Turquie

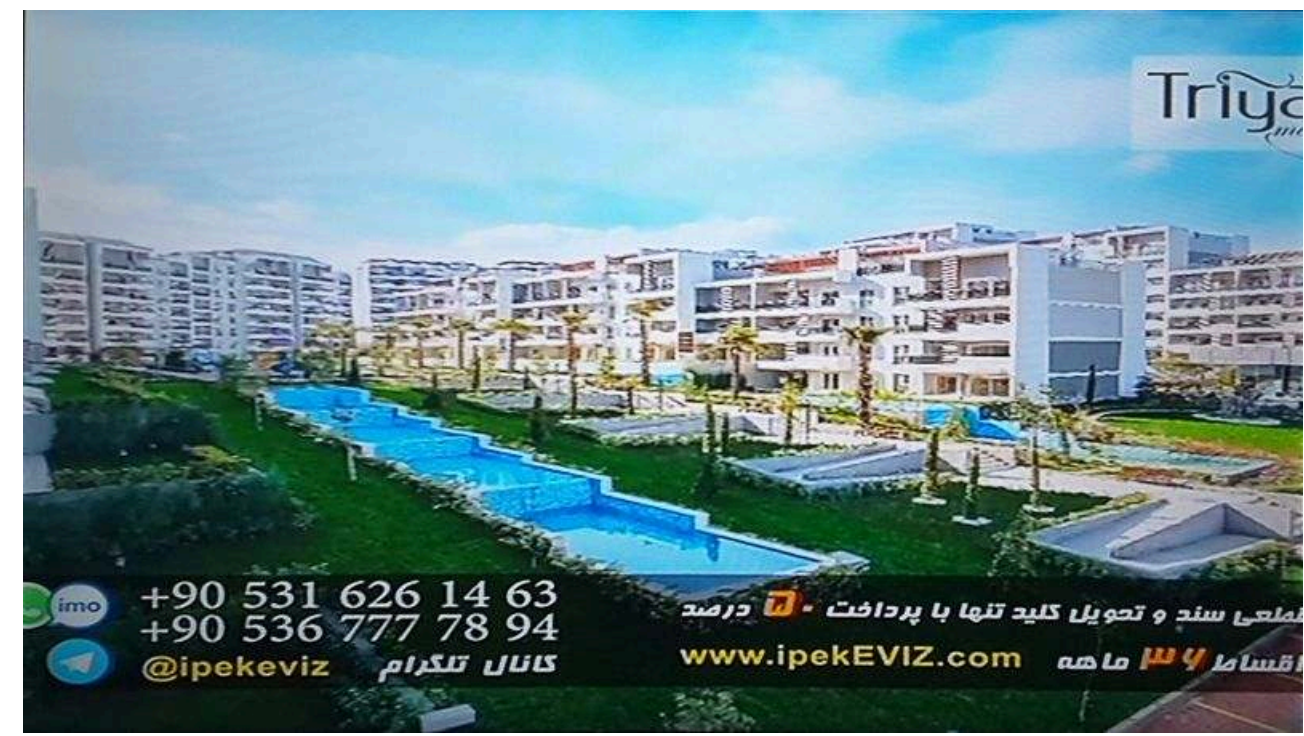

Image publicitaire pour la vente des appartements en Turquie, incluant une offre du crédit et ouvrant aux droits bancaire et de résidence.

Source : GEM TV, capture d'écran Mina Saïdi-Sharouz, août 2018

\section{Les échanges de savoir-faire liés au secteur de la construction}

Historiquement, il y a toujours eu des échanges entre l'Iran et la Turquie dans le domaine de la construction. À la fin des années 1990, les étudiants iraniens en architecture étaient accueillis en Turquie pour leurs études. La Turquie offrait des bourses aux futurs architectes qui travaillaient notamment sur l'histoire de l'architecture de la période Ottomane car une partie des données historiques des Ottomans se trouvait sur le territoire iranien et il y avait une lacune dans la connaissance de ce patrimoine. Les étudiants-chercheurs pouvaient ensuite trouver du travail en Turquie. «Il y a 20 ans, ce sont surtout les Iraniens qui allaient pour travailler dans les ateliers de confection et mais aussi pour travailler dans le bâtiment comme pour les finitions d'enduit et de peinture de bâtiment etc.» nous raconte Mehdi, ingénieur civil, qui poursuit « les Iraniens étaient plus forts dans les finitions. Mais avec l'industrialisation des bâtiments, on a moins besoin de ces services car tout est préfabriqué. Maintenant ce sont les Turcs qui viennent travailler en Iran $»^{33}$. Depuis 2015, des sociétés de construction se sont installées en Iran et ont fait venir des 
ouvriers et des techniciens turcs. Selon les chiffres de l'ambassade de Turquie, plus de 20000 turcs vivent et travaillent de manière permanente en Iran en 2017.

Les importations récentes des produits turcs en Iran favorisent aussi davantage la présence des hommes d'affaires iraniens en Turquie, mais aussi des Turcs en Iran profitant de cette proximité avec les négociateurs et les décideurs locaux pour se placer sur le marché du bâtiment, un secteur clef pour l'économie turque. Une des stratégies adoptées par les constructeurs turcs est de donner à voir en Iran leurs savoir-faire en matière d'exécution et de pilotage des grands travaux. Les constructions pilotes leur servent surtout de vitrine pour exposer ce savoir-faire. Par exemple, depuis 2015, Çumbo, une chaîne alimentaire turque, a ouvert des supermarchés de proximité dans plusieurs quartiers de Téhéran vendant essentiellement des produits turcs. Les magasins sont construits et entièrement équipés par les ouvriers turcs en trois semaines seulement. Les matériaux sont importés de la Turquie. Ce savoir-faire dans la mise en œuvre réalisée en un temps record suscite l'admiration des investisseurs iraniens. Une chaîne d'alimentation indépendante iranienne, nommée Haft, a repris ce concept en ouvrant des supermarchés similaires dans les mêmes quartiers. En copiant le même processus, elle a embauché des techniciens et des gestionnaires turcs.

31 À plus grande échelle, les centres commerciaux turcs peuvent également être considérés comme des modèles pour les investisseurs iraniens ${ }^{34}$. Depuis vingt ans, près de trois cents grands centres commerciaux ont été construits à Téhéran sur le modèle des shopping malls de Turquie et proposent de nombreux produits turcs. Les investisseurs sont iraniens et les crédits viennent des banques locales. Après la levée partielle des sanctions et la volonté d'ouverture de l'économie en Iran depuis 2017, les premiers lieux de commerce turcs ont vu le jour à Téhéran. Cependant, les ventes sont soumises à des conditions imposées par le ministère iranien des Mines, de l'Industrie et du Commerce. Les magasins turcs sont obligés de proposer au minimum $20 \%$ de produits iraniens et, à terme, $100 \%$ des marchandises doivent être fabriquées en Iran ${ }^{35}$.

En juin 2018, un grand centre commercial turc (Turkish Trade center) a été inauguré dans le nord de Téhéran. Ce centre a pour objectif de devenir un grand salon d'exposition mettant en valeur les produits turcs, notamment le prêt-à-porter. Il sert également à démontrer le savoir-faire des entreprises turques dans la construction des méga structures. De grands projets urbains, comme les centres commerciaux Iran Mall (près de deux millions de $\mathrm{m}^{2}$ de surface commerciale) ou le gigantesque complexe de loisir et commerces de Bamland situé dans le $22^{2}$ ème arrondissement à l'ouest de Téhéran, utilisent les matériaux et les accessoires turcs (le bois de synthèse MDF, les charnières, les rails, etc.) pour la construction des bâtiments et pour l'aménagement paysager, identique à celui des séries turques. 
Illustration 12 - Les boutiques turques et l'utilisation des matériaux importés

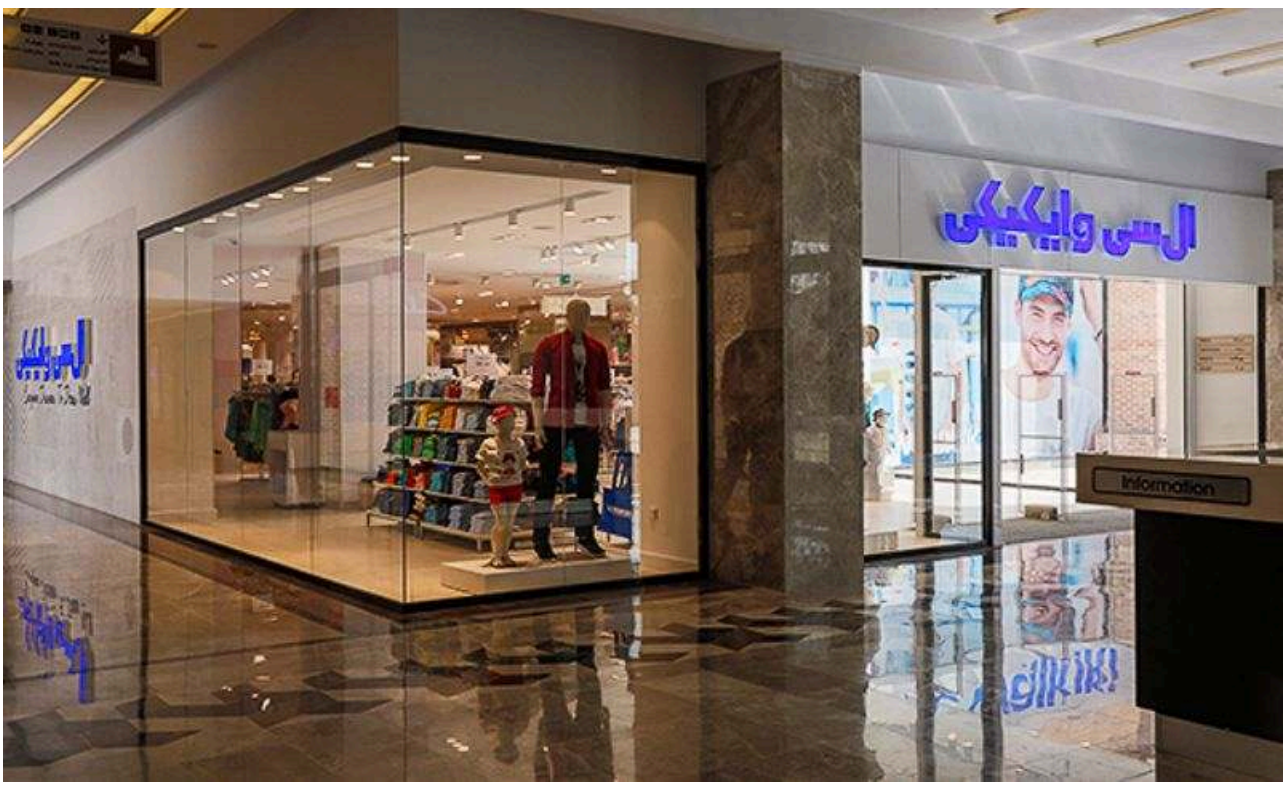

Le centre commercial de Bamland à l'ouest de Téhéran, utilisant les matériaux turcs.

Auteur : Mina Saïdi-Sharouz, novembre 2017.

La foire des matériaux est une autre occasion annuelle pour présenter le savoir-faire turc dans le domaine du bâtiment ${ }^{36}$. L'Iran fournit des pierres et du verre à la Turquie et importe en échange des matériaux comme la robinetterie-plomberie et le MDF utilisés pour la construction des portes et de la décoration intérieure et qui ont conquis le marché ${ }^{37}$. Selon la chambre de commerce, $50 \%$ du bois de synthèse utilisé dans le bâtiment en Iran provient de la Turquie.

\section{Illustration 13 - L'image du Bosphore fréquemment diffusée dans les séries}

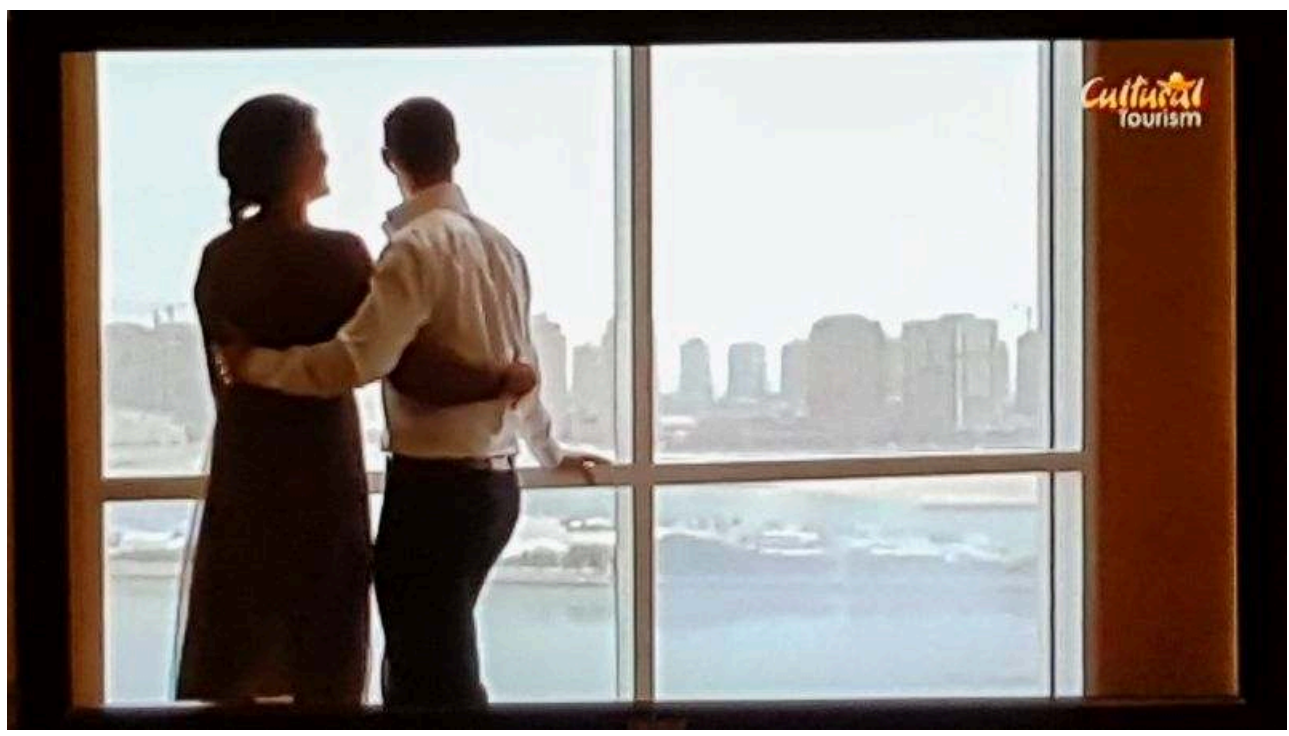

Image tirée d'une série montrant un couple face au Bosphore à Istanbul.

Source : Gem TV. 
Illustration 14 - La vue panoramique sur un lac artificiel de Téhéran pourrait rappeler le Bosphore vu dans les séries

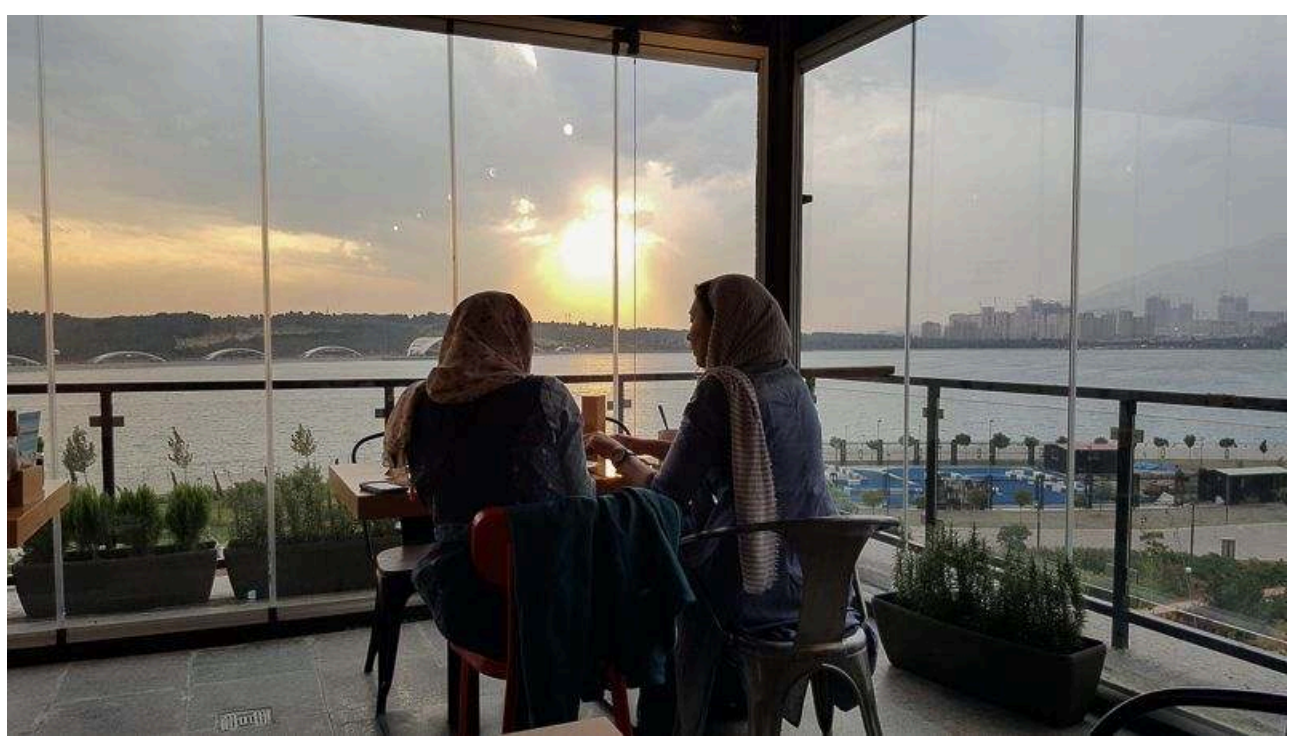

Des jeunes femmes face au lac artificiel de Chitgar, faisant partie d'un grand projet urbain.

Auteur : Mina Saïdi-Sharouz, novembre 2017.

\section{Un modèle turc controversé : le projet immobilier de Pardis} mené par le président Mahmoud Ahmadinejad lors de ses deux mandats (entre 2005 et 2013), a pris comme modèle les grands ensembles construits par $\mathrm{TOKI}^{39}$ autour d'Istanbul. Les opérations de TOKI avaient pour objectif de reloger les habitants des Gecekondu ${ }^{40}$ déplacés suite à la démolition de nombreux quartiers informels abritant les habitants démunis des villes dans les années 2000. Ce projet a rencontré de grandes résistances de la part des habitants et des militants opposés au parti de l'AKP. Selon le géographe Jean-François Pérouse, TOKI s'appuie sur une forte légitimité, due au soutien du président Erdogan, pour mener une politique autoritaire de logement et même plus largement une politique urbaine qui s'inscrit davantage dans une logique ponctuelle, électoraliste ou de marché, que dans une logique d'équité sociale ou d'équité spatiale (Pérouse, 2017).

C'est en suivant ce modèle que la ville nouvelle de Pardis a été conçue à $20 \mathrm{~km}$ au nordest de Téhéran sur le flanc des montagnes d'Elborz sur quatre mille hectares avec l'idée d'accueillir cinq cent mille personnes ${ }^{41}$. La construction a été planifiée sur onze phases. À partir de 2008, la mise en œuvre des premières phases du projet a été confiée à la société de construction turque Cuzo. Les éléments préconstruits constitués de rails et de parois en aluminium, assemblés sur place, sont entièrement importés de la Turquie.

Au démarrage, la société fit venir tous les ouvriers pour garantir la qualité et la vitesse des opérations. Les ouvriers turcs habitaient sur place pendant les travaux. Plus tard, seuls les ouvriers spécialisés dans la menuiserie et le carrelage sont restés et les autres ont été remplacés par des ouvriers iraniens. Pour faciliter la vente, les appartements témoins mettaient en valeur les aménagements intérieurs identiques aux décors des séries turques avec des vues sur un paysage imaginaire (plan d'eau, montagne, etc.), rappelant les images télévisées. Les catalogues donnent à voir un ensemble prestigieux 
d'immeubles fermés par des clôtures et un centre commercial de luxe dans son centre. En attendant, lors de notre dernière visite en janvier 2018, il n'existait encore aucun équipement, ni infrastructure, ni transport en commun dans la zone. Notre enquête montre que les appartements sont plutôt achetés par les ménages appartenant à la classe moyenne, sensibles à l'image prestigieuse de ces appartements et à leurs prix accessibles ${ }^{42}$ et leur solidité grâce à la bonne réputation des constructions turques ${ }^{43}$. "Ceux qui ne peuvent pas acheter en Turquie se contentent des logements à Pardis", me dit une des enquêtées, trentenaire, qui envisage d'acheter un appartement à Pardis. « En plus », ajoute-t-elle, ce n'est pas pollué et pour ma mère qui fait de l'asthme c'est parfait... ».

\section{Illustration 15 - Les modèles turcs de construction de masse reproduits à Istanbul}

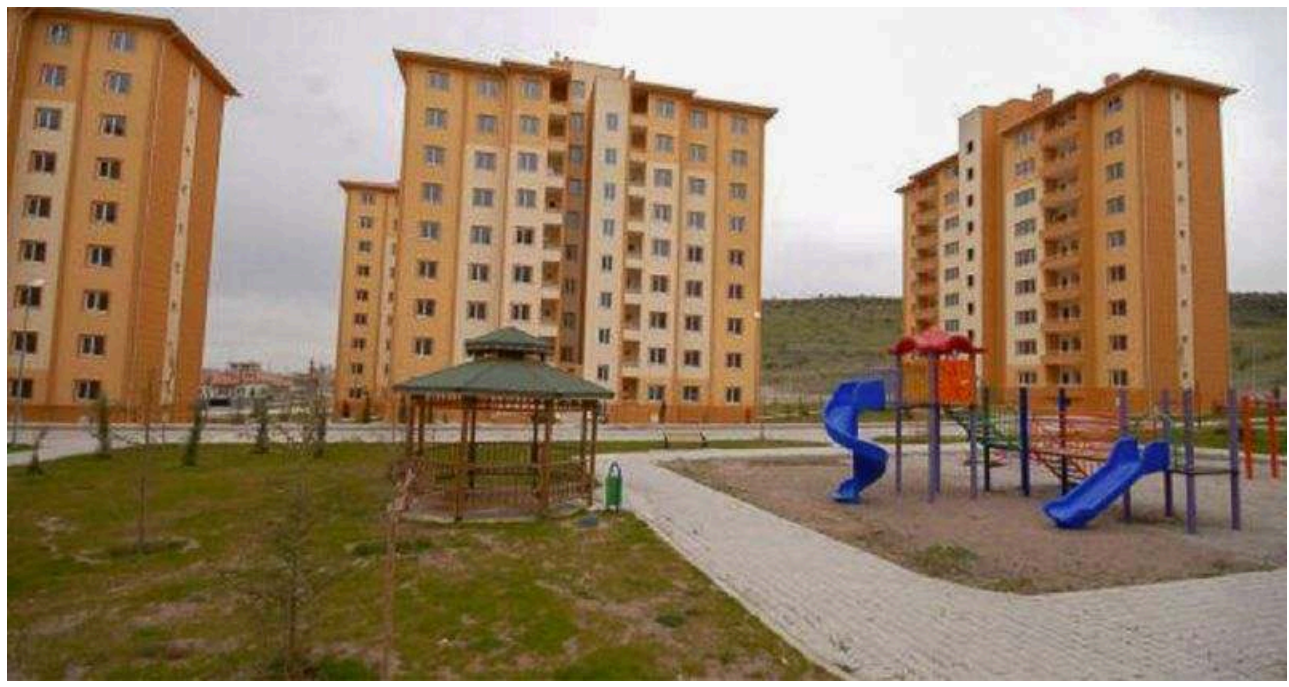

Immeubles construits par TOKI

Source : Site MBD Insaat.com.

Illustration 16 - Les modèles turcs de construction de masse reproduits à Téhéran

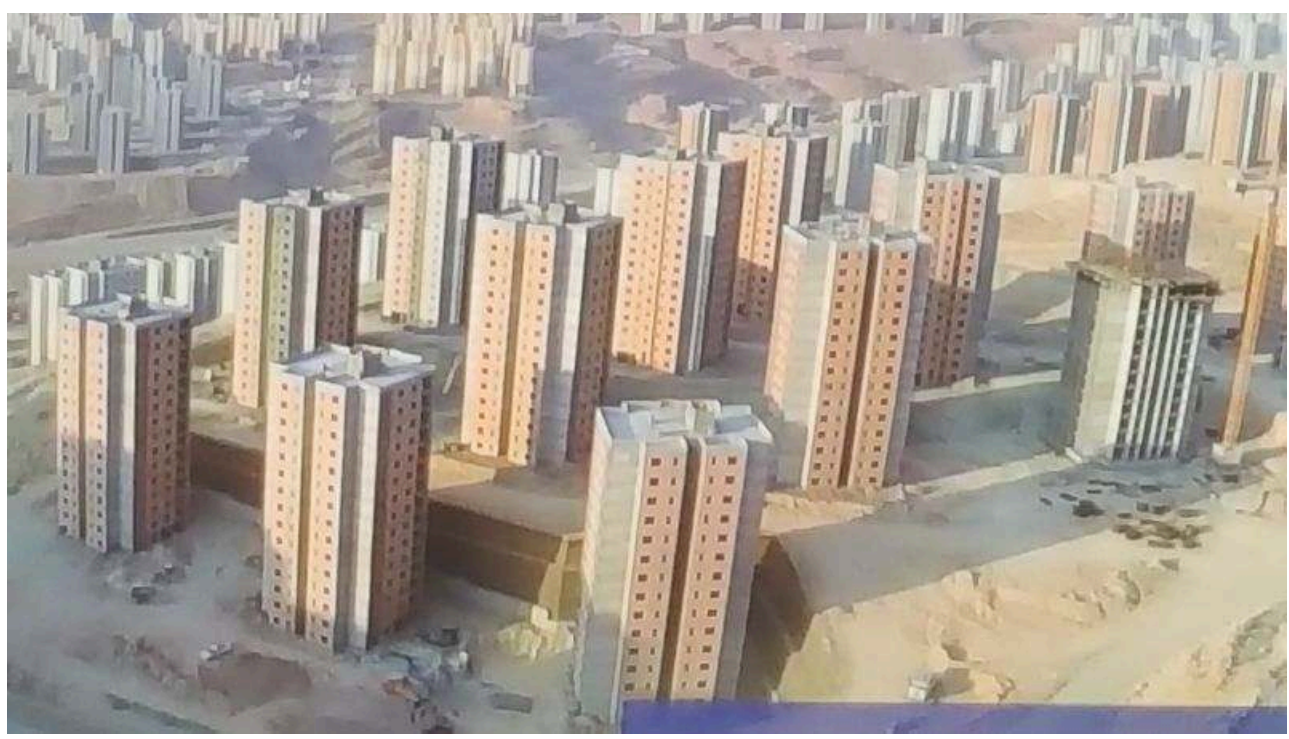

Immeubles construits en partie par la société turque Cuzo à Pardis en périphérie de Téhéran.

Source : Site Ghatreh.com. 
Figure 17 : L'imagerie publicitaire des appartements à Pardis inspirée des décors de séries

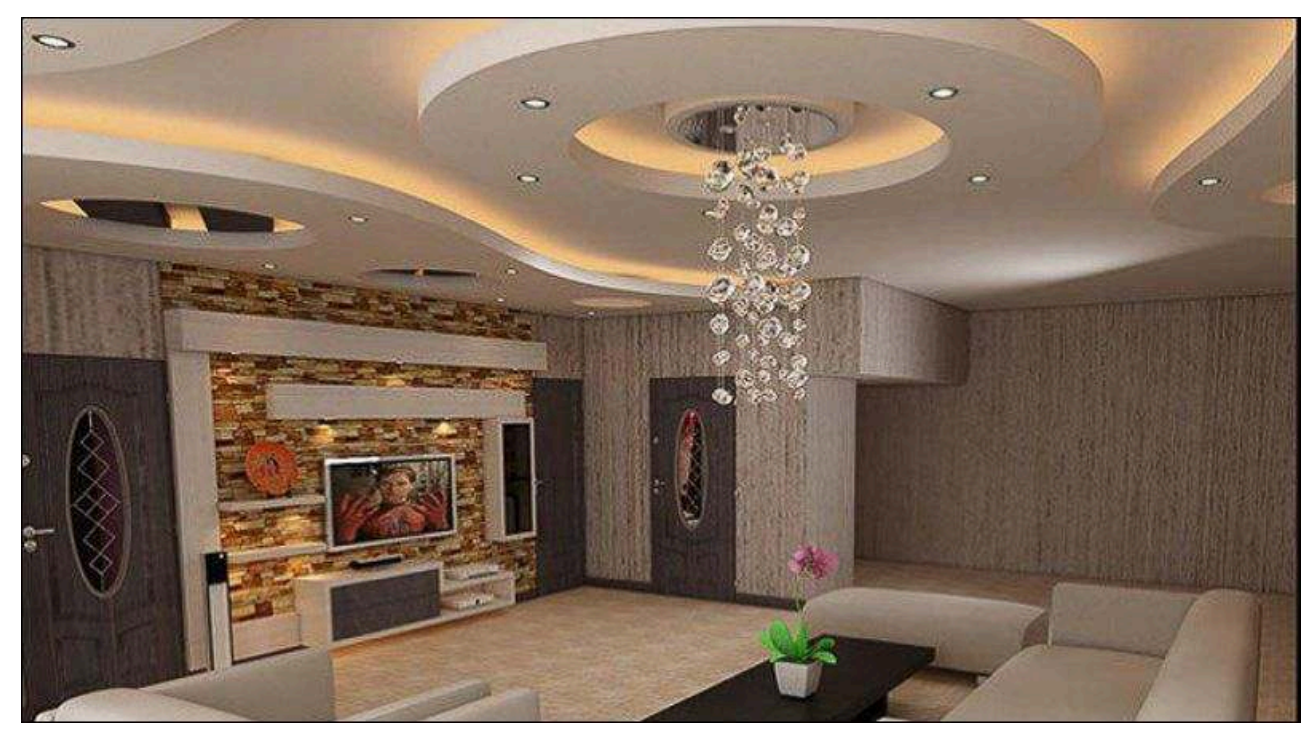

Source : Pardis Niazrooz.com

\section{Conclusion : de la scénographie des séries turques aux grands projets immobiliers}

La réalité, grâce à ces fictions, dépasse notre intuition du départ. Nous avons pu mesurer à quel point les séries turques jouent un rôle important dans la transformation du goût et des modes d'habiter en Iran. Elles contribuent de manière non négligeable à l'ouverture du pays sur l'économie du marché et amplifient les importations turques en Iran.

Grace à la proximité géographique et culturelle des deux pays et à leur rapprochement politique, les Iraniens accèdent au libéralisme économique "par alliance $»^{44}$. En effet, à l'écart de la mondialisation et du fait des sanctions et de plusieurs embargos sur l'Iran, l'économie iranienne s'appuie sur ses relations commerciales avec la Turquie, pour bénéficier, indirectement, de l'économie globale. En quelque sorte, la Turquie devient une porte d'entrée permettant aux Iraniens d'accéder à la globalisation Par mimétisme ou par choix, les Iraniens s'approprient l'esthétique des modèles turcs dans leur vie quotidienne. Grâce à une dynamique commerciale croissante entre les deux pays, ils peuvent ensuite acquérir leurs « objets de désir » sur le marché local. Les produits de luxe ou d'imitation du luxe permettent à la nouvelle classe urbaine de se sentir appartenir au monde global et tenter de sortir de cette double marginalité (être les pauvres d'un pays déjà en marge du processus de la mondialisation) (Sassen, 2009). En revanche, du côté de la Turquie, ces dynamiques contribuent au fonctionnement d'une chaine de production qui dépend en grande partie de l'économie libérale mondiale. Une économie qui s'appuie sur les réseaux informels : d'une part, sur une main-d'œuvre disponible et bon marché fournie par les migrants; d'autre part, sur un système de distribution informelle assumée par les menus commerçants "fourmis voyageurs » (Saïdi-Sharouz, 2015). 
D'autre part, les relations économiques et culturelles très dynamiques entre les deux pays restent dépendantes de la situation politique internationale. Nous constatons qu'avec le retrait des États-Unis de l'accord sur le nucléaire iranien, depuis mai 2018, les Iraniens ont tendance à investir davantage en Turquie, d'autant plus que les deux pays subissent des sanctions de la part des États-Unis et que leurs monnaies respectives ont chuté par rapport au dollar. Le nombre de sociétés iraniennes enregistrées en Turquie est en hausse, ainsi que les investissements dans le domaine de l'immobilier. Selon les statistiques turques, le nombre d'investissements dans l'immobilier par les Iraniens a triplé depuis mai 2018 par rapport à la même période en $2017^{45}$.

Actuellement, le nombre de publicités pour la vente de l'immobilier augmente considérablement lors de la diffusion des séries. La Turquie ouvre ses portes aux Iraniens déstabilisés par la situation internationale. Ils sont de plus en plus nombreux à investir dans l'immobilier avec l'espoir d'acquérir la nationalité turque après cinq ans de résidence et peut-être partir ensuite vers l'Europe. En attendant, la question reste de savoir si ces échanges économiques issus de la circulation de modèles culturels permettront aux Iraniens une véritable ouverture sur le monde, une opportunité pour s'affirmer et pour formuler de nouvelles revendications citoyennes ou au contraire, ils ne vont pas créer des frustrations par des désirs inaccessibles aboutissant à des transferts de capitaux et des départs massifs des Iraniens vers la Turquie?

Quoiqu'il en soit, ces séries et leurs impacts autant sur la vie quotidienne que sur l'économie locale ne cessent de progresser en Iran et semblent être aujourd'hui un phénomène durable qu'il nous appartient de continuer à étudier en ces temps troublés.

\section{BIBLIOGRAPHIE}

Adelkhah F., 2012. Les milles et une frontières de l'Iran. Paris, Kharthala, 492 p.

Aubry D., 2006. Du roman feuilleton à la série télévisuelle. Berne, Peter Lang, 244 p.

Bandura A., Adams N. E., 1977. Analysis of Self Efficacy Theory of Behavioral Change. Cognitive Therapy and Research, Vol. 1, n 4, p. 287-310 [En ligne]. https://www.uky.edu/ eushe2/Bandura/ Bandura1977CTR-Adams.pdf

De Tapia S., 2007. « La circulation des hommes et des biens dans le champ migratoire turc ». In F. Adelkhah \& J.F. Bayart (dir.), Voyages du Développement. Paris, CERI, p. 31-71.

Adelkhah F., 2007. Le réveil du Khorassan. La recomposition d'un espace de circulation. In F. Adelkhah \& J.F. Bayart (dir.), Voyages du Développement. Paris, CERI, p. 115-182.

Esslin M., 1982. The Age of Television. San Francisco, W.H. Freeman \& Co, 138 p.

Girard R., 2008. Mimesis and Theory: Essays on Literature and Criticism, 1953-2005. Palo Alto, Stanford University Press, $344 \mathrm{p}$.

Lipovetsky G., 2015. « Le luxe est un parfait miroir de notre civilisation » Propos recueillis par Denis Lafay. Acteurs de l'Economie. La Tribune [En ligne. https://acteursdeleconomie.latribune.fr/ 
debats/grands-entretiens/2015-05-20/gilles-lipovetsky-le-luxe-est-un-parfait-miroir-de-notrecivilisation.html

Massicard E., 2012. L'AKP de Turquie : héritage islamiste et pratique du pouvoir. In S. Amghar (dir.), Les Islamistes au défi du pouvoir. Paris, Michalon. 196 p.

Compte C., 2013. «L'impact de l'image sur la perception et transformation des représentations mentales », Communication [En ligne], vol. 32, n 1. http://journals.openedition.org/ communication/4842

Nye J., 1990. Bound to Lead: The Changing Nature of American Power. New York, Basic Books, 336 p.

Öktem K., Abou El-Fadl R. (dir.), 2009. Mutual Misunderstandings: Muslims in the European media, Europe in the media of Muslim majority countries. Oxford, European Studies Centre.

Peraldi M., 2016. Le « commerce migratoire » euroméditerranéen. Politique étrangère, vol. $81, \mathrm{n}^{\circ \circ} 4$, p. 35-46.

Perouse J.-F., 2008. Migrations, circulations et mobilités internationales à Istanbul. Studi Emigrazione/Migration Studies, vol. XLV, $\mathrm{n}^{\circ} 172$, p.853-871

Perouse J.-F., 2017. Istanbul planète. Paris, La Découverte, 250 p.

Pinon P., 2006. Les paradoxes de l'occidentalisation de l'architecture à Istanbul à la fin du XIX siècle. Revue romantisme, $\mathrm{n}^{\circ} 131$, p. 51-59

Saïdi-Shaouz M., 2015. Les touristes iraniens à Istanbul. Les "fourmis voyageuses" de la mondialisation. Hommes et migrations, $\mathrm{n}^{\circ} 1312$, p. 81-89. [En ligne]. http://www.hommes-etmigrations.fr/index.php?id=7726

Sassens S., 2009. La globalisation. Une sociologie [traduction française]. Paris, Gallimard, 348 p.

Tarde G., 1993 [1895]. Les lois de l'imitation. [Réimpression de la 2ème édition]. Paris, Kimé Éditeur, $428 \mathrm{p}$.

Tarrius A., 2002. La Mondialisation par le bas. Paris, Balland, 169 p.

Tutal-Cheviron N., Çam A., 2017. La vision turque du "soft-power" et l'instrumentalisation de la culture. In D. Marchetti (dir.), La circulation des productions culturelles. Rabat \& Istanbul, Centre Jacques-Berque, Institut français d'études anatoliennes Casablanca, Centre Jacques-Berque, [En ligne]. https://books.openedition.org/cjb/1226

\section{NOTES}

1. Les sanctions contre l'Iran dont plusieurs embargos ont été mises en place par les États-Unis depuis 1984. En 1984, durant la guerre Iran-Irak, les États-Unis mettent en place un embargo sur les armes contre l'Iran, ainsi que l'interdiction Les autres sanctions dont des embargos sur les armes sont décidés par les Nations unies et l'Union européenne en 2006 et 2007, ainsi que des restrictions sur les services financiers des banques américaines envers l'Iran. Pour rappel, en 1979, suite à la prise d'otages à l'ambassade américaine, les États-Unis gèlent 12 milliards d'actifs financiers détenus par l'Iran.

2. Les Iraniens passent, selon ISNA, le journal universitaire d'Iran, 3 à 4 heures par jours à regarder les séries turques, loin devant les séries indiennes et latino-américaines.

3. Les cinq séries récentes étudiées en profondeur pour cette recherche: Le siècle magnifique (Harim-é Sultan), Bargrizan, L'amour loué (Eshgh-é ejarei), Okeea et La mariée d'Istanbul (Arous-é Istanbuli). 
4. De nouvelles chaînes sont lancées telles que la chaîne de télévision TRT El-Arabiya, en arabe, précédée par TRT 6, en kurde.

5. Turkish Economic and Social Studies Foundation (Anna Lindh Foundation).

6. TRT, TRT 2012 Faaliyet Raporu [Rapport d'activité 2012 de la TRT], Istanbul, 2013 [en ligne] URL :

http://medya.trt.net.tr/medya6/dosya/

2013/07/15/237998f0-4eb4-44ab-9aa2-367da7c6d62d.pdf.

7. La série culte a brisé les records de vente en Turquie avec $59 \%$ des parts de marché lors de sa dernière diffusion en juin 2010.

8. ISPA (Le centre des études sociales universitaires et du sondage d'opinion en Iran) centre des études sociales universitaires.

9. Gem TV diffuse 52 chaînes en persan, une chaîne en kurde, une chaîne en arabe et trois chaînes en turc azéri.

10. GEM Télévision a démarré en 2006, et appartient et est dirigé par le groupe international GEM ("General Entertainment and Media Group").

11. La série Shahrzad réalisée par Hassan Fathi avec 4 saisons est la série la plus populaire en Iran depuis 2015.

12. Nous employons le mot "iranisées", car les séries sont doublées par des voix d'acteurs iraniens en utilisant les expressions iraniennes. Les musiques d'origine sont remplacées par des chansons composées par des artistes iraniens.

13. Khorram Sultan est l'épouse favorite du Suliman le magnifique dans la série Le siècle magnifique, réalisé par Méral Okay.

14. Série réalisée en 2016 par Zeynep Gunay et Deniz Kolos avec la star de la chanson turque Özcan Deniz.

15. On peut retrouver ces successions de styles, selon Pierre Pinon, dans tous les pays de la Méditerranée méridionale ou orientale, que l'on soit dans la situation d'un pays colonisé (Tunis) ou indépendant (Le Caire), au centre d'un empire «oriental» (Istanbul) ou à la périphérie d'un empire européen, mais dont certaines parties ont été ottomanes (Sarajevo).

16. Idem.

17. Hôtel particulier où habitent les grandes familles turques.

18. Série réalisée par Kiralık Aşk, qui a connu un grand succès en Turquie et en Iran. Daphné, une jeune fille du milieu populaire rentre en relation avec Omar, un jeune créateur de mode. Le film donne à voir le milieu de la mode turque et les tensions sociales entre modernité et traditions dans une Turquie en pleine expansion économique. Le film donne à voir de nombreux espaces aménagés de bureaux et d'appartements très modernes.

19. Le samovar est un ensemble de théière et de bouilloire permettant de garder le thé au chaud pendant le temps de son infusion.

20. Karadayi, série télévisée très populaire dont l'histoire se passe dans les années 1970 à Istanbul. La famille Kara vivait heureuse jusqu'à ce qu'un jour, le père, Nazif Kara, soit accusé à tort de meurtre. Mahir Kara fera tout son possible pour prouver que son père est innocent, allant jusqu'à prendre l'identité d'un avocat défunt.

21. Bargrizan est une série dont le scénario est écrit par le grand écrivain turc Reşat Nuri Güntekin. Une famille provinciale émigre à Istanbul et subissent de nombreuses situations involontaires qui changent le destin de chacun des membres de la famille.

22. Résidence fermée.

23. Traditionnellement, chaque objet est acheté en Iran dans les rues spécialisées de la ville ou du bazar de manière séparée. Par exemple, les ménages achètent leurs meubles à Yaftabad, leurs rideaux à la rue Sohravardi et leurs objets de décoration au Grand Bazar. La société Yatas Group a monté depuis 2004 en Iran des boutiques de décoration pour la maison, Enza Home, qui exposent pour la vente l'ensemble des objets d'ameublement et de décoration. Ces magasins ont ouvert 
d'abord sur l'Ile de Kish et puis à Téhéran avec une direction turque et un personnel formé en Turquie.

24. Rasteh ou rue spécialisée est un mode renouvelé de l'organisation spatiale des bazars en Iran. Chaque rue, ou ensemble de rues se spécialise dans la vente d'un type de marchandise. Exemple, le rasteh de livres, le rasteh de chaussures, etc.

25. Les rideaux turcs sont vendus dans les rastehs de rideaux. Les magasins vendent à la fois des rideaux iraniens et des rideaux étrangers. Mais les rideaux turcs sont les plus vendus parmi la classe moyenne et jeunes de Téhéran.

26. L'industrie turque est passée depuis les années 2000 à la production de masse sous l'influence de l'Europe. 600000 centres d'ameublement existent en Turquie! Avec une main-d'œuvre migrante moins chère, les concepteurs sont aussi cosmopolites. En 1998-2000, la part de l'investissement étranger était à $75 \%$. La matière première était aussi importée. Ils ont augmenté la production et ont eu une politique d'exportation. À partir de 2007, la Turquie est devenue un grand pays d'exportation. Aujourd'hui, l'exportation des meubles est une activité très rentable et ramène des devises en Turquie (l'espace d'exportation s'est étendu dans les pays émergents mais aussi jusqu'en Europe, avec, par exemple, la marque Seraye).

27. Cette nouvelle zone d'activité est située sur la route de Jajeroud, à $15 \mathrm{~km}$ à l'est de Téhéran.

28. La notion du modèle turc est employée ici comme un mode d'ameublement contemporain fabriqué.

29. En 2017, suite aux critiques sévères de l'Etat iranien envers les publicités des produits étrangers, la chaîne GEM diffuse également des publicités pour des produits iraniens.

30. Ces boutiques restent très restreintes en nombre mais elles présentent les différents modèles turcs.

31. Les Iraniens ne peuvent pas ouvrir un compte à l'étranger ni avoir une carte de crédit, depuis les sanctions économiques contre l'Iran.

32. En Turquie, les prix de l'immobilier sont relativement bas comparés à d'autres pays européens. Les tarifs abordables, son intention d'intégrer l'UE et son climat ensoleillé sont mis en valeur par les publicités télévisées (une chambre à coucher pour $70000 \$$ ou une luxueuse villa avec vue sur la mer pour $1500000 \$$, selon les publicités).

33. Entretien avec Mehdi, quarantenaire, architecte et homme d'affaire iranien travaillant avec les sociétés turques. Octobre 2017 à Téhéran.

34. Laleh parc (2012), grand complexe de shopping, d'hôtels etc. $\left(91000 \mathrm{~m}^{2}\right)$, dont un étage est All in all. Mais comme cela ne décollait pas, ils ont construit des supermarchés de proximité Cumbo dans les quartiers.

35. Le site officiel du Ministère des Mines, de l'Industrie et du Commerce

36. Une foire des matériaux est organisée chaque année à Téhéran en août. En 2017 il y a eu 1000 exposants iraniens et 200 exposants étrangers dont la plupart des turcs (à la foire du prêt-àporter il y avait 100 exposants dont 34 étaient turcs).

37. Beaucoup de produits européens sont fabriqués en Turquie mais restent plus chers. Par exemple, les portes industrielles italiennes sont produites en Turquie à moitié prix.

38. Maskane mehr: littéralement "les logements d'affection» est le grand projet de logement social de Mahmoud Ahmadinejad, l'ancien président d'Iran.

39. TOKI (Toplu Konut Idraesi), l'agence publique pour la construction de logement,

40. Les Gecekondu (en turc) sont des quartiers d'installations informelles construits par des migrants et de la couche défavorisée, autour des grandes villes de la Turquie, dont Istanbul.

41. La ville nouvelle de Pardis est conçue dans les années 1990. Le projet a été transformé en partie par le programme de logements de masse bon marché "Maskan-é Mehr " (logements d'amour) du président Ahmadinéjad depuis 2007. Ce projet a suscité beaucoup de critiques de la part de la société civile dans les journaux locaux, qui considère ces grands projets comme une 
menace contre les ressources naturelles et la répartition non équitable de l'eau dans la région. De plus, il est critiqué pour le manque d'infrastructures et le non-respect des normes d'urbanisme.

42. Un appartement de $87 \mathrm{~m}^{2}$ se vendait en 2017 à 10000 euros, alors que la même surface avec les mêmes prestations intérieures à Téhéran serait vendue environ six fois plus cher.

43. La publicité des logements turcs met en avant la solidité et la résistance de la structure contre les séismes.

44. « Par alliance ", à l'image d'un membre de la famille par alliance.

45. Cela représente 940 logements selon les statistiques turques.

\section{RÉSUMÉS}

Nous examinons dans cet article, le rôle des séries turques à grand succès, interdites en Iran mais diffusées par satellite (telles que Le siècle magnifique, L'amour interdit et Bargrizan) dans le transfert des modèles urbains et des modes d'habiter en Iran. Comment les séries turques contribuent d'abord au développement de tourisme, puis à l'importation informelle du prêt à porter et enfin à l'ouverture du marché iraniens sur les produits d'ameublement et d'architecture, véritable pôle économique qui transforme les modes d'habiter et agit progressivement sur les espaces des villes iraniennes.

In this article, we examine the role of the very successful Turkish series, no allowed in Iran but broadcast by satellite (such as The Magnificent Century, Forbidden Love and Bargrizan) in the transfer of urban models and ways of living in Iran. How the Turkish series contribute first to the development of tourism, then to the informal importation of the prêt-à-porter and finally to the opening of the Iranian market on the products of furniture and architecture, real economic pole which transforms the modes to live and acts gradually on the spaces of Iranian cities.

\section{INDEX}

Mots-clés : série télévisée turque, Iran, mode d'habiter, architecture intérieure, transfert de modèle urbain

Thèmes : Sur le Champ - Sur le Terrain

\section{AUTEUR}

\section{MINA SAIIDI-SHAROUZ}

Mina Saïdi-Sharouz, minasaidi@yahoo.com, est architecte et géographe. Enseignante à l'École Nationale Supérieure d'Architecture de Paris-La Villette (ENSAPLV), elle est membre du Laboratoire Architecture Anthropologie (LAVUE/CNRS). Elle a publié récemment : - Saïdi-Sharouz M., 2018. L'intégration des migrants dans le centre de Téhéran. La communauté des Ramshehi à Cyrus. In Raulin A., Parsapajouh S. et Blanc-Chaléard M.C. (dir.), Ces villes-là. Actualité de Colette Pétonnet. Paris, Presses Universitaires de Paris Nanterre, p.135-158.

- Saïdi-Sharouz M., 2015. « Les touristes iraniens à Istanbul, les fourmis voyageuses de la 
mondialisation ». Hommes \& Migrations, 2015/4, n 1312, p. 81-89.

- Saïdi-Sharouz M. (dir.), 2013. Le Téhéran des quartiers populaires. Transformation urbaine et société civile en République Islamique. Paris, Karthala-IFRI, 265 p. 